\title{
Inhibition of the Autophagy Pathway Synergistically Potentiates the Cytotoxic Activity of Givinostat (ITF2357) on Human Glioblastoma Cancer Stem Cells
}

\author{
Francesca Angeletti ${ }^{1}$, Gianluca Fossati ${ }^{2}$, Alessandra Pattarozzi ${ }^{3}$, Roberto Würth ${ }^{3}$, \\ Agnese Solari ${ }^{3}$, Antonio Daga ${ }^{4}$, Irene Masiello ${ }^{1}$, Federica Barbieri ${ }^{3}$, Tullio Florio ${ }^{3 * \dagger}$ and \\ Sergio Comincini ${ }^{\text {*十 }}$
}

\begin{abstract}
${ }^{1}$ Department of Biology and Biotechnology, University of Pavia, Pavia, Italy, ${ }^{2}$ Preclinical Research Department Italfarmaco Research Center, Italfarmaco S.p.A, Cinisello Balsamo, Italy, ${ }^{3}$ Department of Internal Medicine, Centre of Excellence for Biomedical Research, University of Genova, Genova, Italy, ${ }^{4}$ Regenerative Medicine, IRCCS Azienda Ospedaliera Universitaria San Martino - IST, Genova, Italy
\end{abstract}

OPEN ACCESS

Edited by:

Jean-Marc Taymans,

French Institute of Health and Medical

Research (INSERM), France

Reviewed by:

Claudia Manzoni,

University of Reading, UK

Manoj B. Menon,

Hannover Medical School, Germany

${ }^{*}$ Correspondence:

Sergio Comincini

sergio.comincini@unipv.it

Tullio Florio

tullio.florio@unige.it

${ }^{\dagger}$ These authors have contributed equally to this work.

Received: 18 July 2016 Accepted: 07 October 2016 Published: 27 October 2016

Citation:

Angeletti F, Fossati G, Pattarozzi A, Würth R, Solari A, Daga A, Masiello I, Barbieri F, Florio $T$ and Comincini $S$ (2016) Inhibition of the Autophagy Pathway Synergistically Potentiates the Cytotoxic Activity of Givinostat (ITF2357) on Human Glioblastoma

Cancer Stem Cells.

Front. Mol. Neurosci. 9:107. doi: 10.3389/fnmol.2016.00107
Increasing evidence highlighted the role of cancer stem cells (CSCs) in the development of tumor resistance to therapy, particularly in glioblastoma (GBM). Therefore, the development of new therapies, specifically directed against GBM CSCs, constitutes an important research avenue. Considering the extended range of cancer-related pathways modulated by histone acetylation/deacetylation processes, we studied the anti-proliferative and pro-apoptotic efficacy of givinostat (GVS), a pan-histone deacetylase inhibitor, on cell cultures enriched in CSCs, isolated from nine human GBMs. We report that GVS induced a significant reduction of viability and self-renewal ability in all GBM CSC cultures; conversely, GVS exposure did not cause a significant cytotoxic activity toward differentiated GBM cells and normal mesenchymal human stem cells. Analyzing the cellular and molecular mechanisms involved, we demonstrated that GVS affected CSC viability through the activation of programmed cell death pathways. In particular, a marked stimulation of macroautophagy was observed after GVS treatment. To understand the functional link between GVS treatment and autophagy activation, different genetic and pharmacological interfering strategies were used. We show that the up-regulation of the autophagy process, obtained by deprivation of growth factors, induced a reduction of CSC sensitivity to GVS, while the pharmacological inhibition of the autophagy pathway and the silencing of the key autophagy gene ATG7, increased the cell death rate induced by GVS. Altogether these findings suggest that autophagy represents a pro-survival mechanism activated by GBM CSCs to counteract the efficacy of the anti-proliferative activity of GVS. In conclusion, we demonstrate that GVS is a novel pharmacological tool able to target GBM CSC viability and its efficacy can be enhanced by autophagy inhibitory strategies.

Keywords: glioblastoma multiforme, programmed cell death, histone deacetylase inhibitor, cancer stem cell, autophagy 


\section{INTRODUCTION}

Glioblastoma (GBM) is fatal, highly invasive brain tumor still displaying poor prognosis (Ohgaki and Kleihues, 2013) even after aggressive multi-modal therapy, including neurosurgery, radiotherapy, and chemotherapy with temozolomide (Stupp et al., 2005). Different GBM features are responsible for the therapeutic failure: the peculiar structure of the brain and the invasive behavior of GBM prevent complete surgical tumor excision; the brain blood barrier prevents systemically administered chemotherapeutics to reach the central nervous system (CNS) in clinically effective concentrations (Omuro and Deangelis, 2013), the molecular complexity of GBM showing a variety of genetic alterations, strongly influencing the therapy outcome, since different mutations might determine different drug sensitivity (Brennan et al., 2013; Patel et al., 2014). In addition, the presence of cancer stem cells (CSCs) within the tumor mass is responsible for the recurrence after therapy (Vescovi et al., 2006; Florio and Barbieri, 2012). CSC theory proposes that tumor development is dependent on a small cell population endowed with selfrenewal and multilineage differentiation ability, a strong resistance to conventional chemotherapy, and the ability to propagate the tumor when xenografted in animal models (Wurth et al., 2014). Since the most aggressive or refractory cancers contain the highest number of CSCs, the therapeutic importance of the eradication of this particular cancer cell subpopulation is a relevant research goal to overcome GBM therapy resistance (Al-Hajj et al., 2004; Singh et al., 2004).

The wide variety of genetic alterations in GBM necessitates the use of strategies targeting processes like chromatin remodeling, which can revert the altered status of multiple genes. The wide range of cellular and molecular effects mediated by histone deacetylase inhibitors (HDACi) make these drugs suitable candidates for the treatment of such heterogeneous tumors (Lee et al., 2015). HDACi act through the inhibition of histone deacetylases, enzymes that control chromatin remodeling and acetylation of histone and non-histone proteins (Ververis and Karagiannis, 2012). Pharmacologicallyinduced unbalanced acetylation (histone acetyl transferase activity) and deacetylation (HDAC activity) in favor of the former, creates an hyperacetylated status, promotes a relaxed chromatin structure and an accessible DNA backbone for the transcriptional machinery favoring the activation/repression of gene transcription (Peart et al., 2005), by which HDACi induce cell death (Bolden et al., 2013), cell cycle arrest, senescence (Pazolli et al., 2012), differentiation, or autophagy (Robert et al., 2011) in tumor cells. In GBM cells, HDACi reduce proliferation via cell cycle arrest and apoptosis, suppress tumor growth in experimental in vivo models, and potentiate the effects of radiotherapy, cytotoxic agents and immune-therapeutics (Thurn et al., 2011). Several HDACi, including SAHA, trichostatin A, valproic acid, belinostat, have been tested in GBM models, and several clinical trials, based on HDACi monotherapy or as drug association strategies are concluded or ongoing (De Souza and Chatterji, 2015).
We report the efficacy of givinostat (GVS), a pan-histone deacetylase inhibitor, on human GBM CSC viability and selfrenewal and the involvement of apoptosis and macroautophagy (hereafter referred as autophagy) in this response.

\section{MATERIALS AND METHODS}

\section{Tumor Samples, Cell Cultures, and Chemicals}

Nine glioma post-surgical specimens were obtained from the Neurosurgery Department of the IRCCS-AOU San Martino IST, (Genova, Italy) after patients' informed consent and Institutional Ethical Committee approval. All patients underwent surgery for the first time and never received chemo- or radio-therapy. Tumors were derived from 6 males and 3 females and the mean age was 57.5 years. Pathological analysis classified gliomas as grade IV glioblastoma $(n=8)$, or grade III anaplastic astrocytoma $(n=1)$ according to World Health Organization criteria. Cell cultures deriving for each tumor sample were coded as GBM1 to GBM9. Patients and tumors details are reported in Supplementary Table 1.

All GBM-derived CSCs were previously isolated and characterized (Gatti et al., 2013; Wurth et al., 2013). Tumor samples were immediately processed to obtain cell cultures enriched in CSCs. Briefly, cell suspension obtained after mechanical dissociation, was filtered through a $40 \mu \mathrm{m}$ strainer (BD Biosciences, San Jose, CA, USA) to remove aggregates, and cultivated in serum-free medium containing DMEMF12/Neurobasal (1:1), B27 supplement (Gibco-Thermofisher, Paysley, UK), 2 mM L-glutamine (Lonza, Basel, Switzerland), $1 \%$ penicillin-streptomycin (Lonza), $15 \mu \mathrm{g} / \mathrm{ml}$ insulin (SigmaAldrich, St.Louis, MO, USA), $2 \mu \mathrm{g} / \mathrm{ml}$ heparin (Sigma-Aldrich) and completed with recombinant human bFGF (10 ng/ml; Miltenyi Biotec, Cologne, Germany) and EGF (20 ng/ml; Miltenyi Biotec) (Bajetto et al., 2013). This medium is defined as "complete medium." These cells gave rise to floating tumorspheres after 2 weeks, but can also growing as stem cells in monolayer, after spheres disaggregation and in presence of Matrigel (BD Biosciences, San Jose, CA, USA), without losing expression of stem cell markers, spherogenic properties, differentiation and tumorigenic potential (Griffero et al., 2009).

All the cell cultures analyzed in this study were previously characterized for tumor-initiating capacity by orthotopic xenograft, induced by injection of 10,000 sphere-derived cells in 6-8-weeks old non-obese diabetic severe combined immunodeficient (NOD/SCID) mice (Charles River Laboratories, Wilminglon, MA, USA), as detailed in previous studies (Carra et al., 2013; Gritti et al., 2014; Corsaro et al., 2016). Animals were housed in pathogenic-free conditions, and handled in agreement with the institutional and national guidelines for the care and use of laboratory animals (Italian D.lgs 26/2014); the experimental plan was approved by the IRCCS AOU S. Martino-IST (Genova, Italy) Institutional Animal Care and Use Committee (IACUC).

To induce differentiation, GBM CSC cultures were seeded and maintained for 2 weeks in DMEM/F12 supplemented 
with $2 \mathrm{mM}$ L-glutamine, penicillin-streptomycin and 10\% FBS (Euroclone, Milano, Italy). Deprivation of growth factors was induced removing bFGF, EGF, and the B27 supplement from the culture medium.

Three human GBM established cell lines were also used: T98G, U373-MG, and U138-MG (ATCC). GBM cell lines were grown in DMEM supplemented with $2 \mathrm{mM}$ L-glutamine, penicillinstreptomycin and $10 \%$ FBS.

Human umbilical cords $(n=2)$ were collected from full-term women, immediately after cesarean section at the Gynecology Department of International Evangelical Hospital (Genova, Italy), after informed consent and approval by Institutional Ethic Committee. After vessel removal, cords were digested with collagenase I-S $(0.5 \mu \mathrm{g} / \mathrm{ml}$, Sigma-Aldrich) for $1 \mathrm{~h}$ to expose Wharton-Jelly and isolated cells cultured in DMEM (10\% FBS, 2 mM L-Glutamine). MSCs were used after full characterization by flow cytometry (MSC Phenotyping Kit, Miltenyi Biotec), as defined by International Society for Cellular Therapy (Dominici et al., 2006).

The pan-HDAC inhibitor givinostat (ITF2357) was kindly provided by Italfarmaco S.p.A. (Cinisello Balsamo, Italy). Givinostat was dissolved in DMSO at the stock concentration of $10 \mathrm{mM}$ and for all experiments it was diluted in the specific medium to obtain the final concentrations. Rapamycin (Cell Signaling Technology, Danvers, MA, USA) and bafilomycinA1 (Sigma-Aldrich), were used to promote the induction and inhibition of autophagy, respectively. Both were dissolved in DMSO at the stock concentration of $100 \mu \mathrm{M}$, and for all experiments drugs were diluted in the specific medium to obtain the appropriate concentration. Controls received the same amount of residual DMSO (not exceeding $0.1 \%$ ) than treated samples.

\section{Sphere-Formation Assay}

GBM CSCs were seeded in complete medium without Matrigel, in 48 -well plates at 1000 cells/well. After $24 \mathrm{~h}$ cells were exposed to increasing concentration of GVS $(0.1-2 \mu \mathrm{M})$, and sphereformation capacity was monitored after 7 days, to allow spheres generation. The number of spheres in wells was quantified using a digital camera mounted on a transmitted light microscope and visually calculated by three independent operators. To further demonstrate the inhibitory activity of GVS on sphereformation process, GBM-derived tumor-spheres, generated in the absence or presence of GVS $(0.1-0.5 \mu \mathrm{M})$, were disaggregated and re-plated in fresh medium avoid of GVS. Spheres-formation efficiency (SFE) (Wurth et al., 2016), calculated as the number of formed spheres/1000 plated cells, was re-evaluated after 7 days.

\section{MTT and Trypan-Blue Dye Exclusion Assays}

Mitochondrial activity, as index of cell viability, was evaluated by measuring the reduction of 3-(4,5-dimethylthiazol-2-yl)2,5-diphenyltetrazolium bromide (MTT, Sigma-Aldrich,). GBM CSCs were plated into 96 or 48-well plates (pre-coated with Matrigel), and the number of cells/well (ranging from 1000 to 5000) was adjusted depending on different proliferation rates and time of treatment. At the end of each treatment, cells were incubated with MTT solution $(2.5 \mathrm{mg} / \mathrm{ml})$, for $2 \mathrm{~h}$. After MTT removal, the formed purple formazan crystals were dissolved in DMSO, and absorbance measured at $570 \mathrm{~nm}$ wavelength (Pattarozzi et al., 2008).

Trypan blue exclusion assay was used to evaluate cell viability reduction induced by GVS treatment. Initially, 5000 cells/well were seeded in 24-well plates, pre-coated with Matrigel. After $24 \mathrm{~h}$, cells were treated with increasing concentration of GVS for additionally 48, 72, and $96 \mathrm{~h}$. Every $24 \mathrm{~h}$, viable cells were counted in the presence of Trypan blue $0.4 \% \mathrm{w} / \mathrm{v}$ (Bio-Rad, MarneslaCoquette, France), to distinguish between dead and live cells, using the TC-20 ${ }^{\circledR}$ automated cell counter (Bio-Rad) (Villa et al., 2016).

\section{Drug Synergism Evaluation}

To determine potential synergistic drug effects on growth of CSCs GBM1, GBM2, and GBM3, cells were exposed to various combinations of GVS $(0.5 \mu \mathrm{M})$ and bafilomycin-A1 (1.25-50 $\mathrm{nM})$. Cell viability was determined by MTT assay after 48 and $72 \mathrm{~h}$ of treatment. Drug interactions (synergistic, additive, antagonistic) were determined by the median effect analysis method, as described (Chou and Talalay, 1984; Chou, 2010), using the CompuSyn software (ComboSyn Inc., Paramus, NJ, USA). This approach takes into account the potency, the shape, and the slope of the dose-dependent neutralization curve of each drug alone and in combination, to calculate a combination index (CI). A CI value of 1 indicates an additive effect, $<1$ indicates synergism, and $>1$ indicates antagonism.

\section{Annexin V/PI Double Staining}

GBM CSCs were treated with GVS and bafilomycin-A1 alone or in association for 48 or $72 \mathrm{~h}$, depending on the experiments. After the treatment, cells were washed gently with PBS, trypsinized, pelleted, secondly washed in PBS and finally counted. According to Annexin V-FITC Apoptosis detection Kit (eBioscience, Hatfield, UK), cells were resuspended in the Annexin Binding Buffer at the concentration of $2-5 \times 10^{5}$ cells $/ \mathrm{ml}$, then $5 \mu \mathrm{l}$ of Annexin-V was added to $195 \mu \mathrm{L}$ of the cell suspension and incubated for $15 \mathrm{~min}$, at the room temperature and protected from direct light. Propidium Iodide $(20 \mu \mathrm{g} / \mathrm{ml})$ was added and cells were analyzed by flow cytometry (FACSCanto II flow cytometer, BD Biosciences) recording 10,000 events per sample. Annexin V-positive cells were considered in the early stages of apoptosis, whereas cells in the late stages of apoptosis were Annexin V- and PI-positive (Carra et al., 2013).

\section{Real-Time PCR Expression Analysis}

Total RNA from GBM CSCs was extracted using the Aurum Total RNA Mini Kit (Bio-Rad), according to the manufacturer's instruction, and reverse transcribed into cDNA using the iScript cDNA Synthesis Kit (Bio-Rad). Single stranded cDNA products were analyzed by Real-time PCR using the SsoFast ${ }^{\mathrm{TM}}$ Eva Green Supermix (Bio-Rad,) on a CFX96 Touch Real-time PCR (Bio-Rad). Cycling conditions were set at $94^{\circ} \mathrm{C}$ for $30 \mathrm{~s}$, $60^{\circ} \mathrm{C}$ for $30 \mathrm{~s}$ and $72^{\circ} \mathrm{C}$ for $30 \mathrm{~s}$, for 37 cycles. Primers, for MAP1LC3B amplification, were pre-designed by PrimePCR (BioRad). Human HPRT1 and TBP pre-designed primers (Bio-Rad) 
were used as internal controls. Levels of target genes in each sample were normalized on the basis of HPRT1 and TBP amplification and reported as relative values (Mathur, 2014).

\section{Immunoblotting Analysis}

Following specific treatments, cells were lysed in buffer containing 1\% Igepal, $20 \mathrm{mM}$ Tris- $\mathrm{HCl}, \mathrm{pH} 8,137 \mathrm{mM} \mathrm{NaCl}$, $10 \%$ glycerol, 2 mM EDTA, $1 \mathrm{mM}$ phenylmethylsulfonyl fluoride, $1 \mathrm{mM}$ sodium orthovanadate, $10 \mathrm{mM} \mathrm{NaF}$ (all from SigmaAldrich), and the "Cømplete protease inhibitor mixture" (Roche) for $20 \mathrm{~min}$ at $4^{\circ} \mathrm{C}$. Nuclei were removed through centrifugation (5000 rpm at $4^{\circ} \mathrm{C}$ for $10 \mathrm{~min}$ ); total protein concentration was measured with Bradford assay (Bio-Rad). Proteins (40$60 \mu \mathrm{g})$ were resuspended in Laemmli buffer (2\% SDS, 62.5 $\mathrm{mM}$ Tris, $\mathrm{pH} 6.8,0.01 \%$ bromophenol blue, $1.43 \mathrm{mM} \beta 2$ mercaptoethanol,and $0.1 \%$ glycerol) and were separated on 10 or $12 \%$ (depending on protein size) SDS-PAGE, and subsequently transferred onto PVDF membrane (Bio-Rad) (Massa et al., 2004). The following antibodies were used: anti-LC3B, anti-Beclin1, anti-Atg7, and anti-acetyl- $\alpha$-tubulin (Lys40) (all from Cell Signaling Technologies). $\beta$-actin (Cell Signaling Technologies) and $\alpha$-tubulin (Sigma Aldrich) were used as internal control to ensure equal loading and transfer of proteins. Antibodies were all diluted at 1:1000 in 3\% bovine serum albumin (BSA), except $\alpha$-tubulin which was diluted 1:7500 in Tween $20(0.1 \%) /$ PBS. Species-specific peroxidase-linked ECL secondary antibodies (GE Healthcare USA, 1:5000 dilutions) were used. Protein signals and densitometric analysis were performed using the Clarity Western ECL substrate (BioRad) and the Chemi-Doc System (Bio-Rad).

\section{LC3B-GFP Autophagosome and Electron Microscopy Analysis}

For autophagosome detection, GBM CSCs were seeded at the density of 5000 cells/well in 48 -well plates pre-coated with Matrigel. After $12 \mathrm{~h}$, cells were transduced with BacMam LC3B-GFP viral particles (multiplicity of infection, MOI = 30), according to the Premo Autophagy Sensor Kit (Invitrogen, Carlsbad, CA, USA). After $16 \mathrm{~h}$ of incubation, cells were treated with GVS $(0.5 \mu \mathrm{M})$, bafilomycin-A1 $(10 \mathrm{nM}$, added $4 \mathrm{~h}$ before the end of the time interval), the combination of the two drugs, or vehicle (controls); LC3B-GFP signals were monitored after additional $24 \mathrm{~h}$, using an inverted fluorescence microscope (40X magnification, Eclipse Nikon TS100, Minato, Japan). GFPpositive vesicles were analyzed in number and shape (area) using the Autocounter tool as described (Fassina et al., 2012).

For ultrastructural analysis, GBM CSCs were grown at $75 \%$ confluence, and treated with GVS $(0.5 \mu \mathrm{M})$ or vehicle. Samples were prepared according to Marchesi et al. (2014). Specifically, after $48 \mathrm{~h}$ p.t, cells were harvested by centrifugation at 800 rpm for $3 \mathrm{~min}$ and fixed with $2 \%$ glutaraldehyde in medium, maintained for $2 \mathrm{~h}$ at room temperature. Cells were then rinsed in $\mathrm{PBS}$ ( $\mathrm{pH}$ 7.2) overnight and post-fixed in $1 \%$ aqueous $\mathrm{OsO}_{4}$ for 2 $\mathrm{h}$ at room temperature. Cells were pre-embedded in $2 \%$ agarose in water, dehydrated in acetone, and finally embedded in epoxy resin (Electron Microscopy Sciences, EM-bed812). Ultrathin sections (50-60 nm) were collected on formvar-carbon-coated nickel grids and stained with uranyl acetate and lead citrate. The specimens were finally observed with a Zeiss EM900 electron microscope equipped with a $30 \mu \mathrm{m}$ objective aperture and operating at $80 \mathrm{kV}$.

\section{Cell Transfection and Atg7 Silencing}

To modulate Atg7 protein expression, GBM2 CSCs (80\% confluence) were transiently transfected with Lipofectamine LTX reagent (Invitrogen) in presence of pooled Silencer select validated siATG7 sequences (s20650, s20651; Ambion, USA) at the final concentration of $150 \mathrm{nM}$ or with the mock solution alone, as negative control. To perform the viability assay (MTT), cells were plated in 96-well plates (2000 cells/well) and assayed after 24,48 , and $72 \mathrm{~h}$ p.t. For protein lysates, transfection was performed on cells seeded on $60 \mathrm{~mm}$ petri dishes with the same concentration of pooled siATG7 sequences.

\section{Statistical Analysis}

All reported experiments were carried out in triplicate and performed at least three times. Data were reported as means \pm SEM. All statistical analysis (ANOVA followed by Dunnett's posthoc test, or unpaired two-tailed Student's $t$-test), were calculated with Graph-Pad Prism 5.0. $p \leq 0.05$ was considered statistically significant.

\section{RESULTS}

\section{GVS Affects the Viability of GBM Cell Lines and CSC Enriched Cultures}

Different established human GBM cell lines (i.e., U87-MG, U138MG, and T98G) were initially tested to evaluate the effect of GVS on cell viability, using the MTT assay. Time-course (from 24 to $96 \mathrm{~h}$ ) and dose-response (GVS $0.1-2 \mu \mathrm{M}$ ) experiments were performed. GVS reduced cell viability in a concentration- and time-dependent manner (Supplementary Figure 1), although no effects were observed for short time treatments (24h) or GVS concentrations lower than $0.25 \mu \mathrm{M}$.

Due to the higher translational potential of GBM CSCs compared to cell lines (Lee et al., 2006), we confirmed the effect of GVS on the viability of CSC-enriched cultures isolated from nine human GBMs. GVS induced anti-proliferative effects in all CSC cultures grown as monolayers in a concentration- and timedependent manner, with, also in this case, lower efficacy observed for shorter treatments (Figure 1, Supplementary Table 2). The mean GVS IC 50 , calculated after $72 \mathrm{~h}$ of treatment, was $0.85 \mu \mathrm{M}$ but this value decreased proportionally with GVS exposure time, confirming the time-dependent activity of the drug (Table 1).

Growing CSCs as monolayer on Matrigel allows for better cellular and biochemical characterization without interfering with stem-like characteristics (Griffero et al., 2009; Wurth et al., 2013), although the ability to grow as tumor-spheres is a defining feature of these cells. Thus, we verified whether this experimental condition interfere with CSC drug responsivity. Comparing GVS $(0.1-2 \mu \mathrm{M})$ effects on CSCs grown as either tumor-spheres or monolayer, we observed no differences in drug sensitivity (data not shown). 


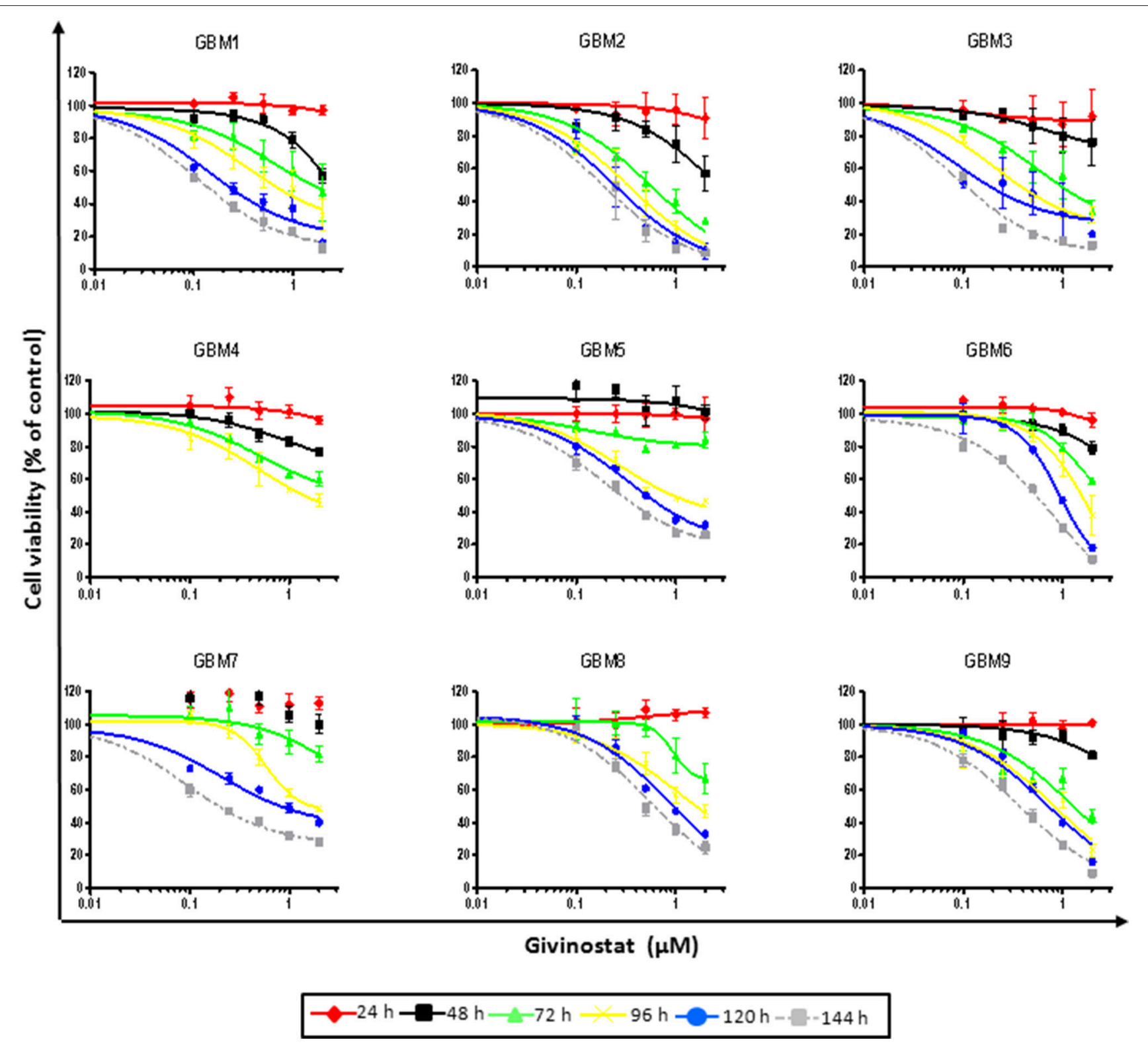

FIGURE 1 | GVS dose-response curves (0.1-2.0 $\mu$ M) in nine adherent GBM cell cultures enriched in CSCs. Cell viability was determined by MTT assay after 24-144 h of treatment. Experiments were performed in triplicate and percentage of inhibition was calculated vs. untreated control cells.

The cellular and molecular mechanisms of GVS activity on GBM CSCs were analyzed in the most responsive CSC cultures (GBM1, GBM2, and/or GBM3) (Table 1), in comparison, in selected experiments, with less responsive cultures (GBM7, GBM8, GBM9). To evaluate the contribution of cytostatic or cytotoxic effects of GVS, GBM1, and GBM2 CSCs were assayed by Trypan blue exclusion test, after treatment with GVS $(0.5-2 \mu \mathrm{M}$ for $48-96 \mathrm{~h})$. Forty-eight hour treatment caused cytostatic effect at all concentrations tested (with the exception of GBM1 treated with high GVS concentration) with no changes in the number of dead cells compared to untreated cells at $\mathrm{T}_{0}$. However, extending treatment time, cells started to die in a concentration- and time-dependent manner (Supplementary Figure 2).

To establish the minimum time of GVS exposure required to decrease CSC viability, we performed cell growth recovery experiments (Favoni et al., 2010). GBM1, GBM2, and GBM3 cultures treated with $0.5 \mu \mathrm{M}$ GVS, were washed and incubated in drug-free fresh medium. Cell viability by MTT assay determined either immediately after medium replacement or after additional 24-72 h (see Supplementary Figure 3 for the experimental scheme). Twenty-four hour treatment decreased growth rate and the low proliferative activity partly persisted during the following $24 \mathrm{~h}$, but after additional recovery time the cells reacquired an 


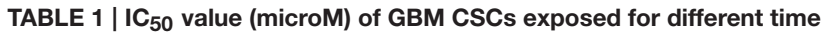
intervals to GVS.

\begin{tabular}{lcccc}
\hline CSCs & $\mathbf{7 2 h}$ & $\mathbf{9 6} \mathbf{h}$ & $\mathbf{1 2 0} \mathbf{h}$ & $\mathbf{1 4 4} \mathbf{h}$ \\
\hline GBM1 & 0.63 & 0.35 & 0.15 & 0.11 \\
GBM2 & 0.55 & 0.33 & 0.24 & 0.19 \\
GBM3 & 0.56 & 0.20 & 0.09 & 0.09 \\
GBM4 & 0.51 & 0.49 & - & - \\
GBM5 & Not reached & 0.28 & 0.32 & 0.19 \\
GBM6 & 1.47 & 0.93 & 0.93 & 0.66 \\
GBM7 & 1.19 & 1.54 & 0.20 & 0.09 \\
GBM8 & 1.23 & 1.01 & 0.89 & 0.57 \\
GBM9 & 1.29 & 0.83 & 0.72 & 0.37 \\
\hline MEAN IC & 0.85 & 0.66 & 0.44 & 0.30 \\
VALUE (RANGE) & $(0.51-1.47)$ & $(0.20-1.54)$ & $(0.09-0.93)$ & $(0.09-0.66)$
\end{tabular}

Calculations were performed using GraphPad Prism software. (Not reached, absence of a significant GVS activity; empty boxes, not performed).

exponential-like growth rate, reaching levels comparable to nontreated cells. The extension of the treatment with GVS to 48 or $72 \mathrm{~h}$ delayed the time to recovery causing, after $72 \mathrm{~h}$ of treatment, a significant reduction in cell viability (Supplementary Figure 4).

\section{GVS Inhibition of Cell Survival Is Specific for CSCs and Is Diminished after Differentiation}

GVS anti-proliferative effect was also tested in differentiated GBM cells obtained by shifting CSC cultures from growth factor-enriched to FBS-containing medium. After 14 days, differentiated GBM CSCs lose in vivo tumorigenicity and stem cell marker expression and exhibit glial and/or neuronal marker expression (Gritti et al., 2014; Banelli et al., 2015). GVS did not affect the viability of differentiated GBM1 and GBM2 cells (hereafter named GBM1 DIFF and GBM2 DIFF), even after $144 \mathrm{~h}$ of treatment with the highest concentration of drug (Supplementary Figure 5A). Furthermore, GVS treatment of two cultures of normal human umbilical cord-derived mesenchymal stem cells (MSCs) caused no reduction of viability at all concentrations and times tested in MSC1, whereas only the highest GVS concentration $(2 \mu \mathrm{M})$ slightly reduced MSC2 viability (Supplementary Figure 5B). These results confirm that independently from the culturing conditions, the antitumoral effects of GVS are selectively directed against CSC specific proliferation mechanisms, with GBM DIFF cells and MSCs mostly insensitive to the drug.

Self-renewal is a defining feature of both normal and cancer stem cells. Tumor-sphere formation is considered an indirect index of self-renewal of CSCs (Soeda et al., 2008). To understand whether GVS affects GBM CSC self-renewal, we performed a spherogenesis assay. GBM1, GBM3, GBM5, GBM7, and GBM9 CSCs were plated at 1000 cells/well in the presence of increasing concentrations of GVS and allowing to generate spheres for 7 days. As reported in Figure 2A (and quantified in Figure 2B), GVS inhibited spherogenesis in a dose-dependent manner. At low GVS concentrations, tumor-spheres were less compact and organized, while a clear reduction of number and size of spheres was observed on increasing the drug concentration. At the highest concentrations tested (GVS 1 and $2 \mu \mathrm{M}$ ), the inhibition of cell viability in all the cultures was predominant and masked the drug effect on spherogenesis, making difficult the interpretation of the results. A statistically significant reduction of the number of spheres was seen in GBM1, GBM3, and GBM9 starting from the GVS concentration of $0.25-0.5 \mu \mathrm{M}$ which had low effect on CSC viability, suggesting that GVS inhibition of sphere formation is independent from anti-proliferative effects. An additional experimental approach was performed using GBM1, GBM2, and GBM3 preformed tumor-spheres, which were treated with low GVS concentrations $(0.1-0.5 \mu \mathrm{M})$ for 7 days, disaggregated and re-plated in GVS-free medium (Figure 3A,B). While untreated CSCs regenerate spheroids, pre-treatment with GVS dosedependently abolished their spherogenesis ability.

\section{Effects of GVS on Apoptosis/Autophagy Processes in GBM CSCs}

GVS was tested for the ability to interfere with apoptosis and autophagy processes. GBM1, GBM2, and GBM3 CSCs, treated with GVS (up to $2 \mu \mathrm{M}$ ) for $72 \mathrm{~h}$, were tested in AnnexinVPI double staining by FACS analysis (Figure 4), showing a concentration-dependent increase of cells entering the early phases of apoptosis (approximately 30\% for GBM1 and GBM3, and $50 \%$ for GBM2).

To study GVS modulation of autophagy, as a preliminary evaluation we analyzed the relative expression of $M A P 1 L C 3 B$, coding for LC3B, in GVS-treated GBM1, GBM2, and GBM3 CSCs by Real-time PCR. GVS treatment induced a transient increase in MAP1LC3B mRNA content (detectable after 24 and $48 \mathrm{~h}$, and back to the baseline after $72 \mathrm{~h}$, Supplementary Figure 6). Then, the effect of GVS on LC3II and Beclin1 protein content was investigated in GBM1, GBM2, GBM3, GBM6, GBM7, and GBM9 cells. CSCs were treated for $72 \mathrm{~h}$ with GVS $(0.5-2 \mu \mathrm{M})$ or with the autophagy inducer rapamycin ( $50 \mathrm{nM}$, for $72 \mathrm{~h}$ ): LC3-II and Beclin 1 showed an increased amount following GVS treatment, often higher than those caused by rapamycin administration (Figure 5). Increased acetyl- $\alpha$-tubulin content, a direct marker of HDAC6 inhibition (Haggarty et al., 2003), was observed following GVS administration, confirming the efficacy of the drug on its specific target (Figure 5).

To verify whether GVS-mediated increase in LC3-II and Beclin1 was the resultant of autophagy activation, GBM1, GBM2, and GBM3 CSCs were transduced with a baculovirus LC3B-GFP expressing vector. Cells were treated with GVS $(0.5 \mu \mathrm{M})$, bafilomycin-A1 $(10 \mathrm{nM})$ or with the combination of the two drugs and analyzed for the amount of GFPpositive vesicles, using the Autocounter tool as described (Fassina et al., 2012). GVS, bafilomycin-A1 and the combined treatment induced significant increases of fluorescent vesicles in the investigated cells; furthermore, the additive results in increasing GFP puncta in the combined GVS+bafilomycin-A1, compared to bafilomycin-A1 alone administration, suggested a direct increase of the autophagy flux induced by GVS 


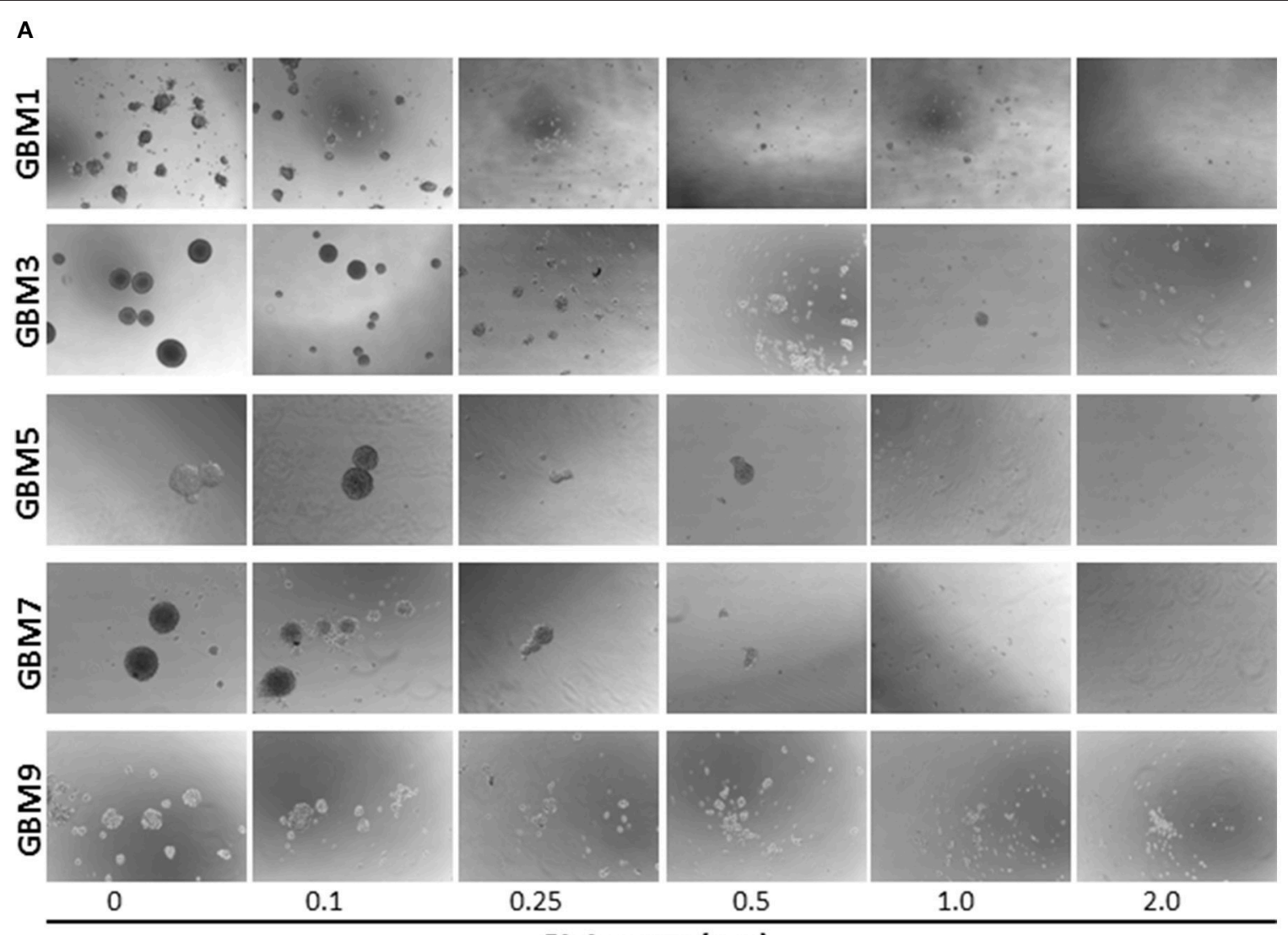

Givinostat ( $\mu \mathrm{m})$

B

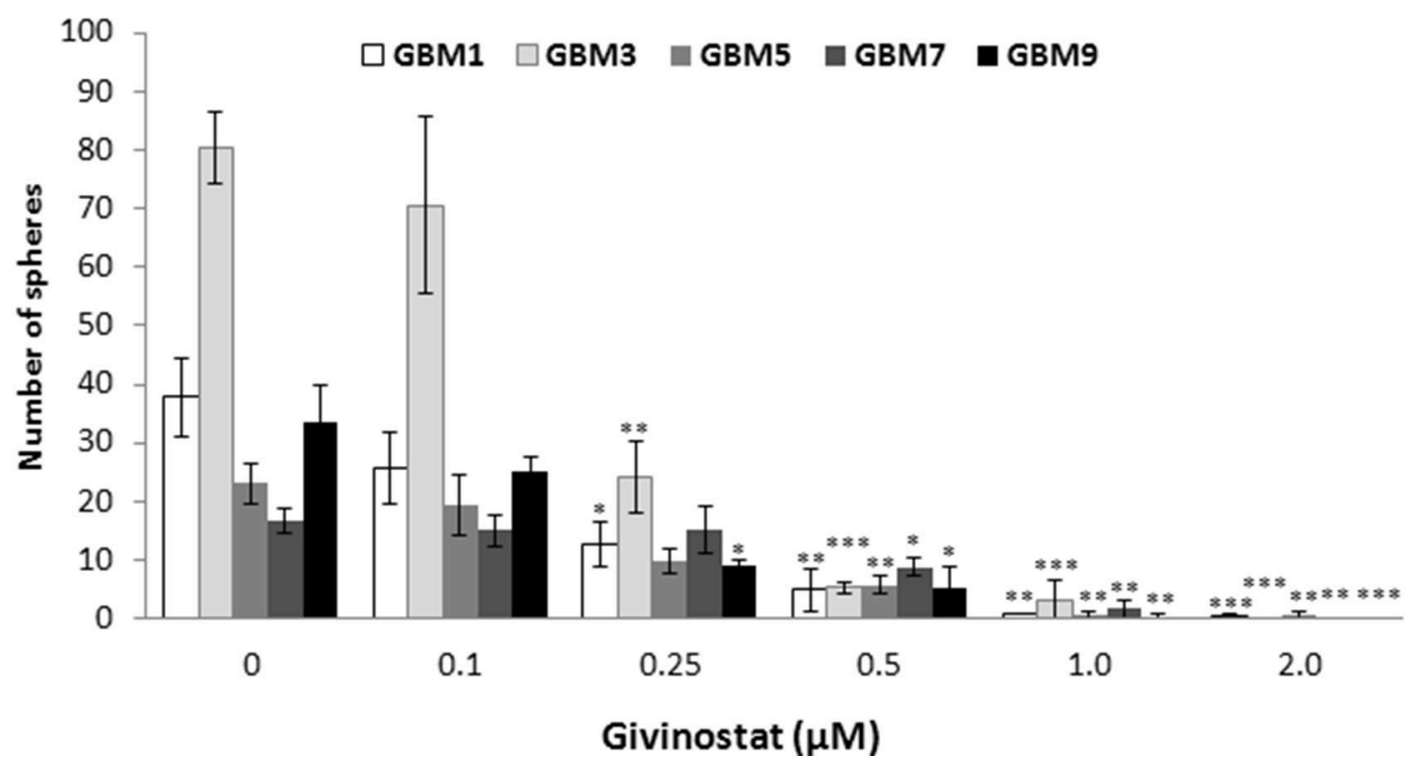

FIGURE 2 | GVS effect on GBM CSC sphere formation. (A) GBM1, GBM3, GBM5, GBM7, and GBM9 were maintained in the selective medium for stem cells in the absence or presence of increasing concentrations of GVS. Sphere formation was visually monitored and after 14 days the number of spheres/well was counted. Representative microphotographs (magnification 10X) are reported. (B) Histogram reports the mean sphere number from four experiments $\left({ }^{*} p<0.05,{ }^{* *} p<0.01\right.$, ${ }^{\star \star \star} p<0.001$, ANOVA followed by Dunnett's post-hoc test). 


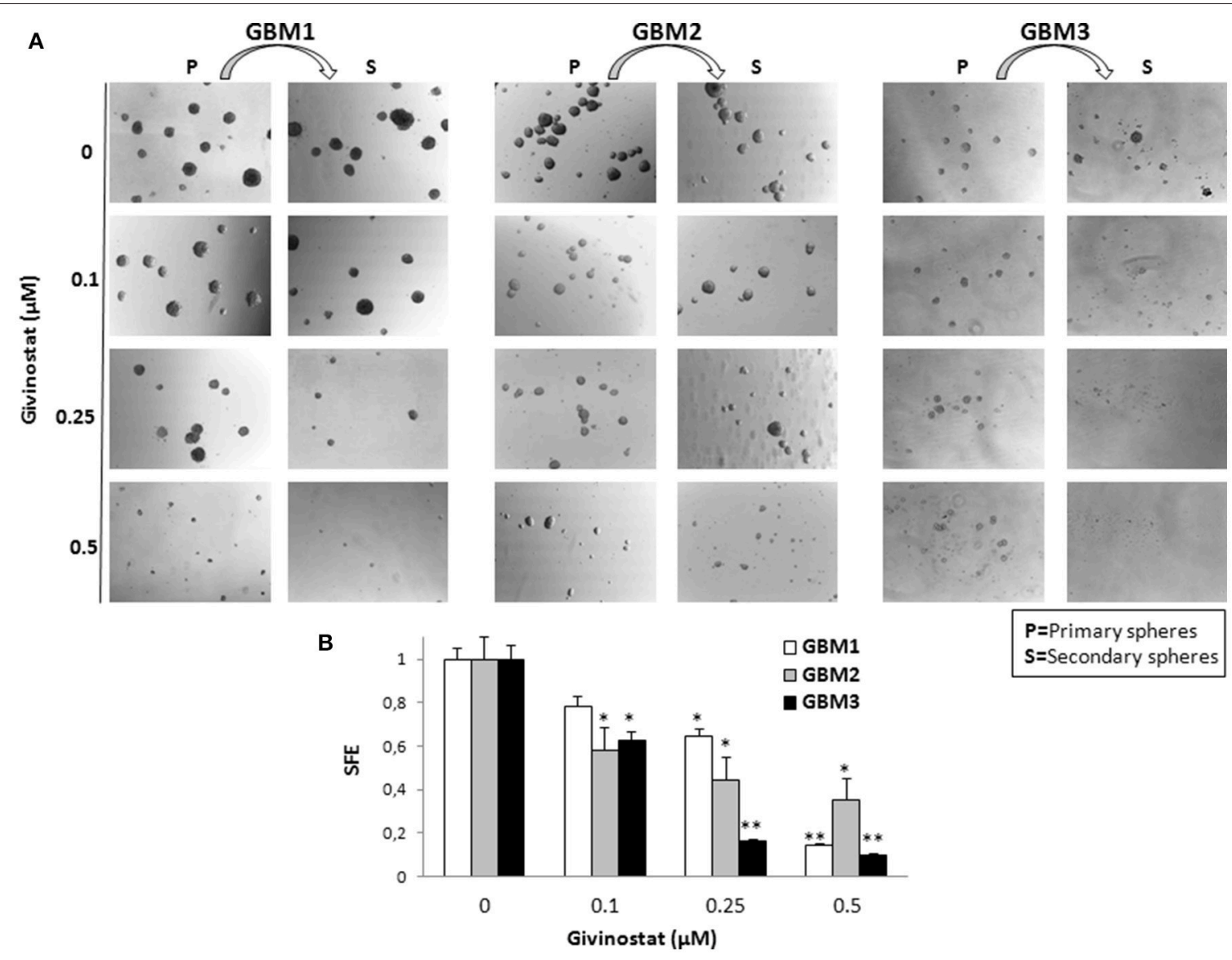

FIGURE 3 | GVS effect on GBM CSCs on self-renewal measured as secondary sphere formation. (A) GBM1, GBM2, and GBM3 were grown in stem cell permissive conditions for 7 days to allow sphere appearance ( $P=$ Primary spheres) in the presence or absence of increasing concentration of GVS. Spheres were then disaggregated and single cells were replated in medium without GVS and, following further 7 days, the formation of secondary spheres (S) was evaluated. Microphotographs (magnification 10X) are representative of GVS inhibitory effect on spherogenesis. (B) Histograms indicate the sphere-formation efficiency (SFE) of secondary spheres (mean number of spheres/number of cells seeded per well) for each condition against untreated controls. Control value was set at $1{ }^{*} p<0.05$ and ${ }^{* *} p<0.01$ using ANOVA followed by Dunnett's post-hoc test).

(Figures 6A,B). Transmission electron microscope analysis of GVS-treated cells, revealed a high number of double membrane autophagic-like vesicles containing partially undigested material. Features of mitophagy (the presence of mitochondria within autophagosomes), multivesicular bodies and phagophores (a site of autophagosome formation) were also scored (Figure 6C).

\section{Functional Role of Autophagy in GVS-Treated GBM CSCs}

The functional significance of the autophagy induced by (or in response to) GVS treatment was assessed using different known autophagy inhibitors/inducers (Klionsky et al., 2016).

To arrest the autophagy process, GBM1, GBM2, and GBM3 CSCs were pre-treated with bafilomycin-A1 (up to $50 \mathrm{nM}$ ) for $2 \mathrm{~h}$ before being incubated with GVS $(0.5 \mu \mathrm{M})$. Cell viability was evaluated by MTT assay. Forty-eight hours of combined treatment with bafilomycin-A1 and GVS induced a significant decrease in cell viability, compared to bafilomycin-A1 or GVS alone (the combination of bafilomycin-A1+GVS reduced GBM1 viability of $57 \%$ compared to $-25 \%$ of bafilomycin-A 1 and $-23 \%$ GVS; in GBM2 the combined treatment caused a reduction of $-74 \%$, in comparison to bafilomycin-A $1-31 \%$ and GVS $-20 \%$; while in GBM3 we observed $-46 \%$ viability after bafilomycinA1+GVS, compared to $-8 \%$ of bafilomycin-A 1 and $-20 \%$ GVS), with a further increase in the cytotoxic effect induced by the combined treatment after $72 \mathrm{~h}$ (Figure 7A). To determine the statistical significance of the GVS/bafilomycin-A1 association, the combination index $(\mathrm{CI})$ values were calculated and plotted using the CompuSyn software (Supplementary Figure 7): CI values above 1 are indicative of antagonism, while below 1 of synergism. GBM1, GBM2, and GBM3 showed CI values $<1$ starting from the combination GVS $0.5 \mu \mathrm{M}$ /bafilomycin-A1 2.5 $\mathrm{nM}$ for GBM1 (range, $0.2-0.6$ at $48 \mathrm{~h} ; 0.07-0.6$ at $72 \mathrm{~h}$ ), GVS 0.5 $\mu \mathrm{M} /$ bafilomycin-A1 $5 \mathrm{nM}$ for GBM2 (range, 0.3-0.9 at $48 \mathrm{~h}, 0.3-$ 0.9 at $72 \mathrm{~h}$ ) and GVS $0.5 \mu \mathrm{M} /$ bafilomycin-A1 $2.5 \mathrm{nM}$ for GBM3 (range: $0.4-0.6$ at $48 \mathrm{~h} ; 0.02-0.4$ at $72 \mathrm{~h}$ ), confirming a synergistic effect exerted by GVS in association with the autophagy inhibitor bafilomycin-A1. Similar results were obtained when the effects of 


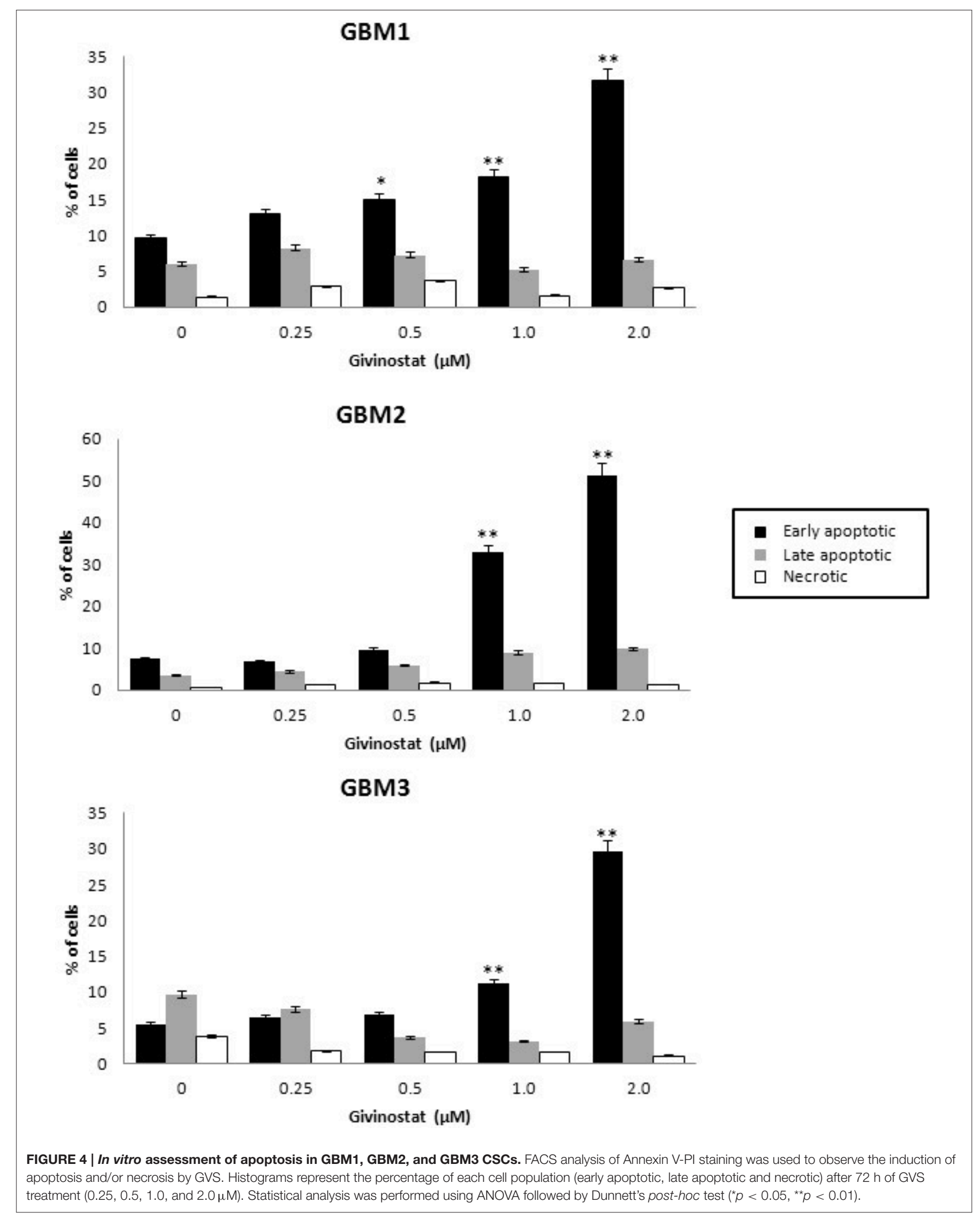




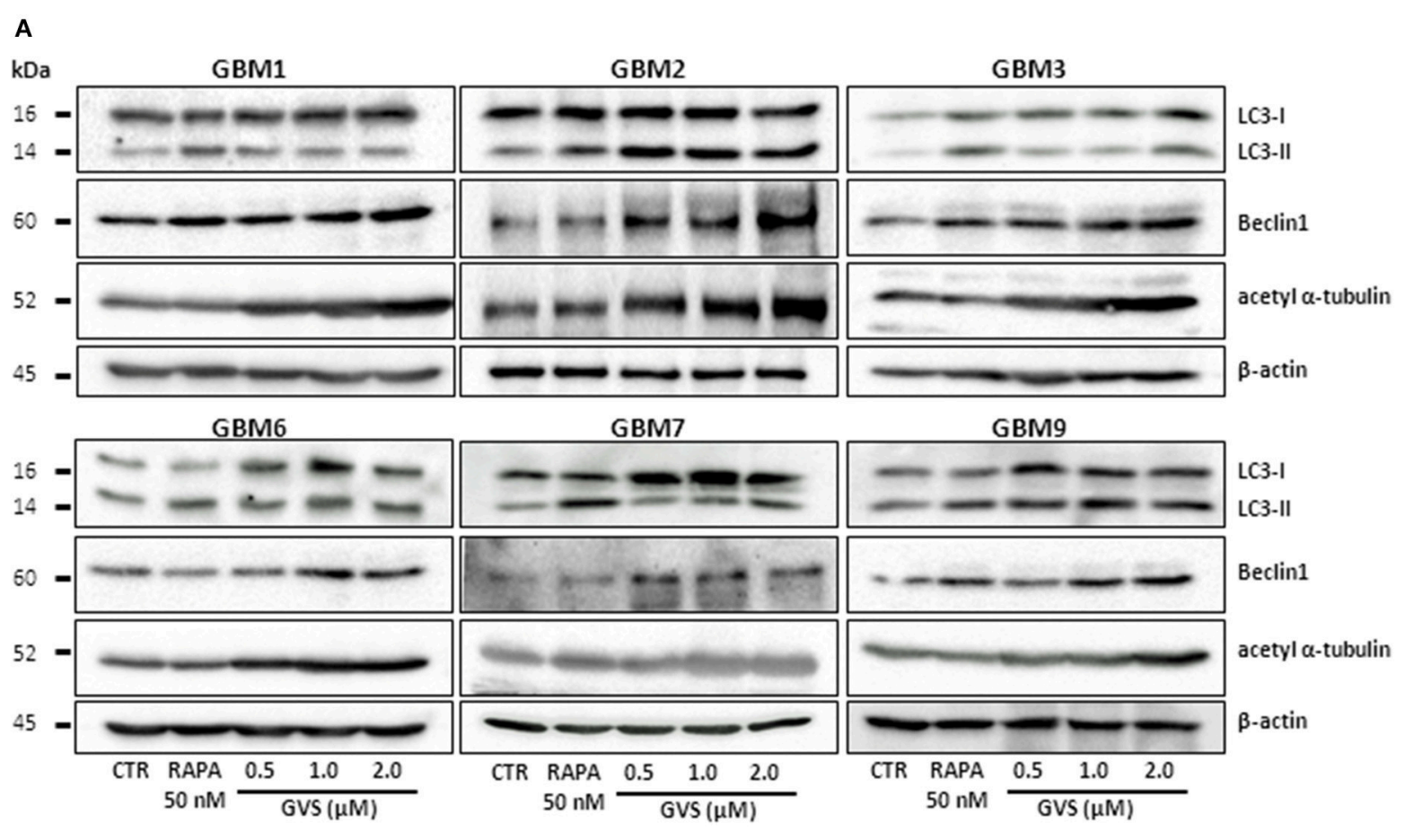

B

GBM1

GBM2

GBM3
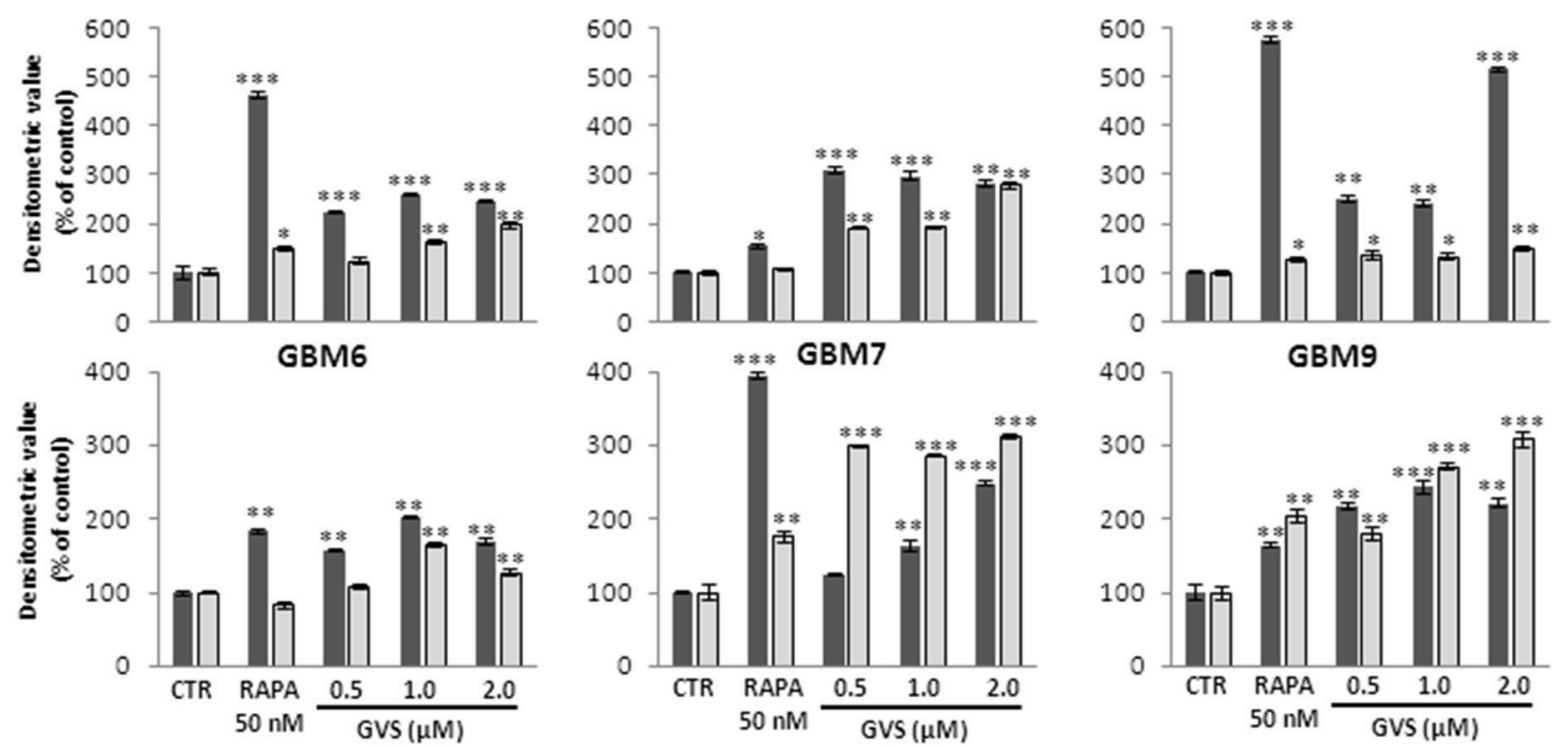

\section{- LC3-II $\square$ Beclin1}

FIGURE 5 | Modulation of autophagic marker expression following GVS administration in GBM1, GBM2, GBM3, GBM6, GBM7, and GBM9 CSCs. (A) LC3-I, LC3-II, Beclin1 and acetyl- $\alpha$-tubulin protein levels was determined by immunoblotting. $\beta$-actin was used as protein loading control. Lysates were obtained from cells treated for $72 \mathrm{~h}$ with GVS $(0.5,1.0$, and $2.0 \mu \mathrm{M})$ or rapamycin $(50 \mathrm{nM})$. (B) Densitometric analysis of protein expression. Values were normalized to $\beta$-actin expression and reported as percentage of untreated controls. Experiments were performed in triplicate, data represent mean $\pm \mathrm{SEM},\left({ }^{*} p<0.05 ;{ }^{* *} p<0.01,{ }^{* \star *} p<\right.$ 0.001, ANOVA followed by Dunnett's post-hoc test). 

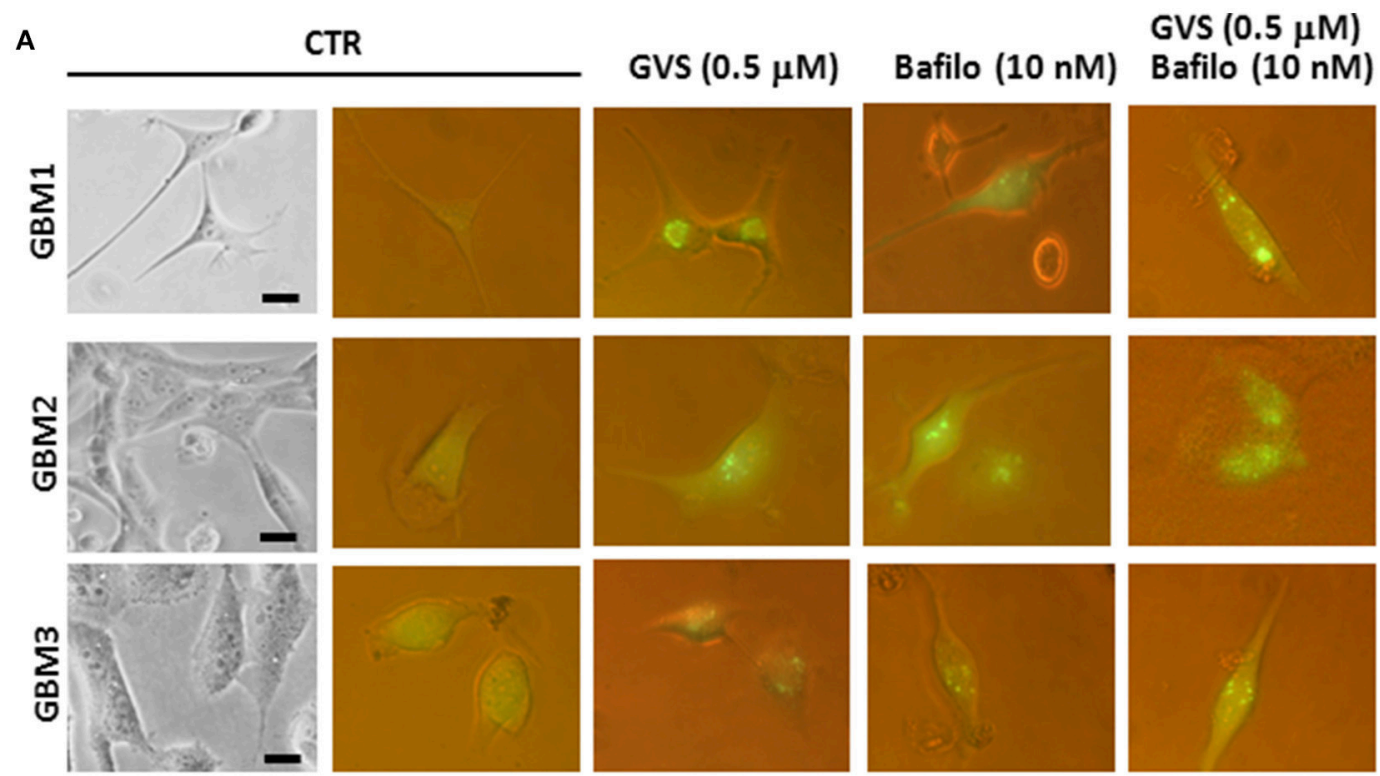

B
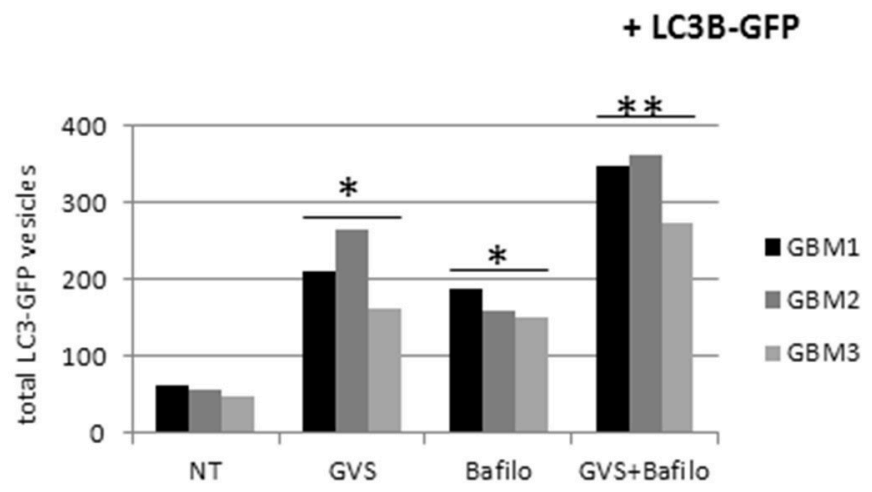

C CTR
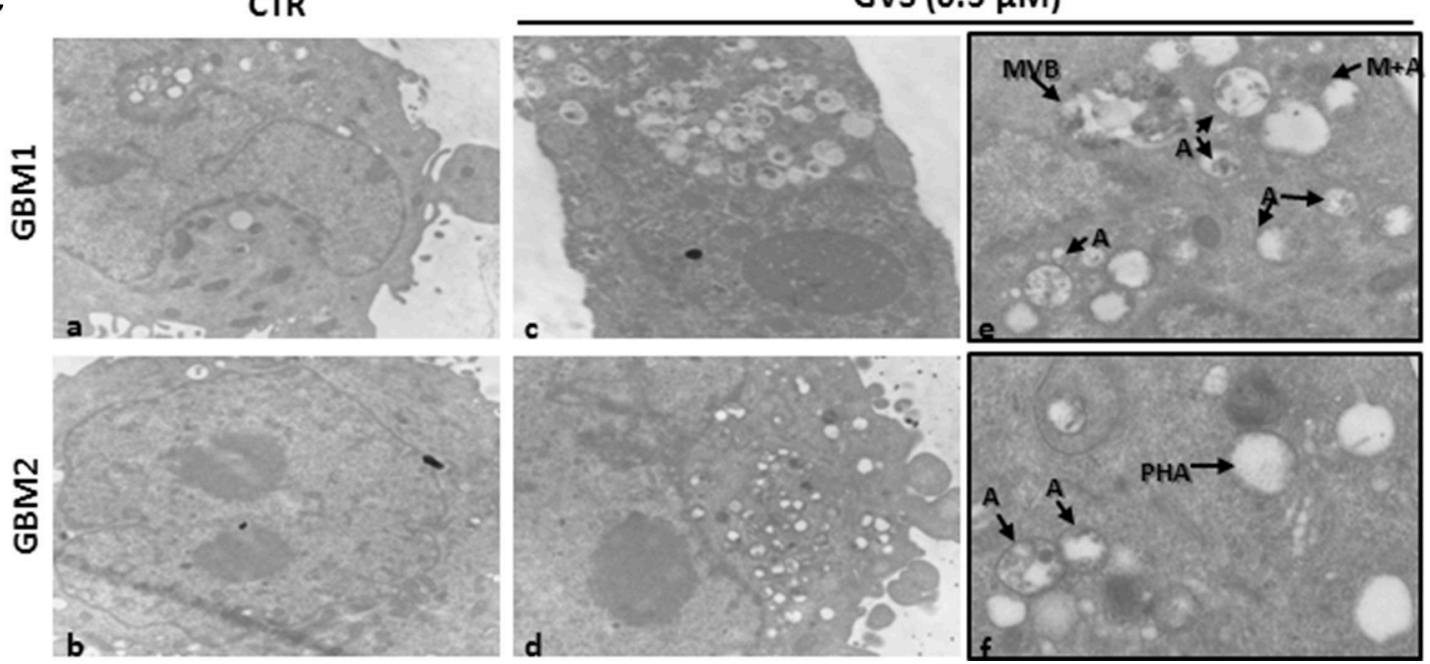

FIGURE 6 | LC3B-GFP expression in GBM CSCs exposed to GVS. (A) Cells were transduced with baculovirus LC3B-GFP expressing vector. GVS (0.5 $\mu$ M) was added $16 \mathrm{~h}$ after transduction to allow LC3B-GFP expression. Bafilomycin-A1 $(10 \mathrm{nM})$, alone or in combination with GVS (0.5 $\mu \mathrm{M})$, was added $4 \mathrm{~h}$ before the end of the $24 \mathrm{~h}$ interval. LC3-GFP signals were monitored using an inverted fluorescence microscope (40X magnification). (B) Evaluation of numbers of LC3-GFP vesicles was performed using Autocounter tool. GFP-positive vesicles were considered with area $>1 \mathrm{~mm}^{2}$ in 50 cells for each treatment. Statistical analysis was performed 


\section{FIGURE 6 | Continued}

using ANOVA followed by Dunnett's post-hoc test ( ${ }^{*} p<0.05,{ }^{* *} p<0.01$ ) comparing GVS, bafilomycin-A1, GVS+bafilomycin-A1 treatments to the respective untreated (CTR) GBM CCSs. (C) Representative ultra-structures analysis using transmission electron microphotographs of GBM1 and GBM2 treated with GVS $(0.5 \mu \mathrm{M})$ for $72 \mathrm{~h}$. GBM1 and GBM2 cells $\left(10^{6}\right)$ were grown at $75 \%$ confluence and treated (c-f) or not (a,b) with GVS. Cells were harvested, fixed and stained for ultra-structural visualization. Specimens were then observed with a Zeiss EM900 electron microscope. Magnifications are 7000X (a-d) and 12000X (e,f). "A" indicates large intracellular vesicles, likely autophagosomes; "M+A" indicates a mitochondrion fused with a vesicle (mitophagy), "MVB" indicates a multivesicular body, finally "PHA" indicates a phagophore (isolation membrane) assembly site.

the combined GVS $(0.5 \mu \mathrm{M}) /$ bafilomycin-A1 (5 nM) treatment was investigated on the induction of apoptosis in GBM2 and GBM3 CSCs. As compared to single drug treatments, the drug combination increased the percentage of apoptotic cells (GBM2: $41.27 \%$ of early+late apoptotic cells with the association, vs. 19.84 and $6.25 \%$ for GVS and bafilomycin-A1, respectively; for GBM3, $16.4 \%$ of early+late apoptotic cells with the association, vs. 5.89 and $6.25 \%$ for GVS and bafilomycin-A1, respectively). Also the percentage of PI-positive, necrotic cells was increased with the combined treatment with GVS and bafilomycin-A1 (Figure 7B). These results suggested that the inhibition of autophagy by bafilomycin-A1 facilitated GVS-dependent apoptotic cell death in GBM CSCs. Furthermore, GVS+bafilomycin-A1 treatment produced an increase of LC3-II and p62 content, as compared to the amount of these proteins following individual drugs treatments. Conversely, in presence of the autophagy-activator rapamycin or with GVS alone, p62 protein levels were reduced as a result of the increase in autophagy-mediated protein degradation (Figures 8A,B).

To better decipher the role of autophagy in GVS antitumor activity, GBM2 CSCs were transfected with siATG7 validated sequences, to arrest autophagy by reducing the intracellular content of Atg7. The efficacy of gene silencing was verified $24 \mathrm{~h}$ post-transfection when Atg7 protein was significantly down-regulated as compared to mock and not-transfected cells (Figure 9, left panel). Treatment of siATG7-GBM2 with GVS caused a further decrease of cell viability (Figure 9, right panel), confirming that the arrest of autophagy enhances GVS tumor cytotoxicity, as also showed by bafilomycin-A1 treatment.

Finally, a widely adopted culture-starvation scheme was applied to activate autophagy in GBM1 and GBM2 CSCs. In detail, CSCs were deprived of growth factors for $60 \mathrm{~h}$ before being treated with GVS (up to $2 \mu \mathrm{M}$ ) and cell viability measured by MTT assay. Deprivation of growth factors prevented GVSdependent cell death, and the activation of autophagy in these conditions was demonstrated by increased LC3-II and reduction in p62 protein levels (Supplementary Figure 8).

\section{DISCUSSION}

In this paper we report that in human GBM stem cells, the inhibition of autophagy using both chemical and RNAi approaches increases the antitumor efficacy of givinostat.

Givinostat (ITF2357, GVS) is a hydroxamic acid-derivative, orally active, pan-HDACi. GVS has anti-inflammatory, antineoplastic and anti-angiogenic properties (Leoni et al., 2005), with pro-apoptotic effects in hepatocellular carcinoma (Armeanu et al., 2005), non-small cells lung cancer (Del Bufalo et al., 2014) and acute myeloid leukemia cells (Galimberti et al., 2010).

We report the efficacy of GVS in reducing viability of human GBM CSCs, the subpopulation responsible for GBM resistance to conventional therapy and patients' fatal outcome (Bonavia et al., 2011; Chen et al., 2012; Florio and Barbieri, 2012). In vitro, CSCs retain genotypic and phenotypic features of the tumor they are derived from, which are progressively lost when the cells are grown in FBS-containing media, as occurs in established cell lines (Lee et al., 2006). Thus, results obtained using CSC-enriched cultures possess a higher traslational impact than those obtained adopting established cell lines. CSC-enriched cultures, obtained from nine human GBMs, were cultured both as spheroids and monolayers retaining the stem-like biological and phenotypical characteristics (expression of stem cell markers, multilineage differentiation, self-renewal, and tumorigenicity in vivo; Griffero et al., 2009; Wurth et al., 2013).

Firstly, we documented that GVS powerfully reduces viability of all CSCs analyzed, independently from the genotypical and phenotypical heterogeneity observed in the GBMs: we observed a prolonged pharmacological activity that could be useful during in vivo experimental schemes. GVS also inhibited CSC self-renewal, a mechanism necessary to preserve CSCs within the tumor microenvironment. It is important to highlight that the culture conditions used to grow CSCs (non-adherent tumor-spheres or monolayers in the presence of Matrigel) did not affect GVS efficacy. In contrast, differentiated GBM cells or normal stem cells are modestly affected by GVS-induced cell death. Accordingly, previous studies reported that GVS is cytotoxic for hepatoma cell lines but not for normal human hepatocytes (Armeanu et al., 2005), and, in agreement with our results, that MSCs are resistant to GVS at concentrations higher than $1 \mu \mathrm{M}$ for at least $72 \mathrm{~h}$ (Golay et al., 2007). The reduced activity against non-tumor stem cells while impairing CSC viability, renders GVS an interesting candidate for further clinical evaluations. In addition, we report that GVS had a lower effect on differentiated CSC, further supporting the selectivity of GVS against CSC subpopulation.

It was reported that antitumor activity of GVS depends on the activation of apoptotic pathways (Pathil et al., 2006; Golay et al., 2007; Galimberti et al., 2010). We report that the effect of GVS on GBM CSC viability is associated not only with apoptotic cell death but also with the activation of macroautophagy. Autophagy is a central cell degradation system involved in several physiological and pathophysiological events (Rabinowitz and White, 2010), including cancer (Mathew et al., 2009; Macintosh and Ryan, 2013). A functional link between HDACi and autophagy was demonstrated in cancer cells (Zhang and Zhong, 2014). To this regard, we evaluated 


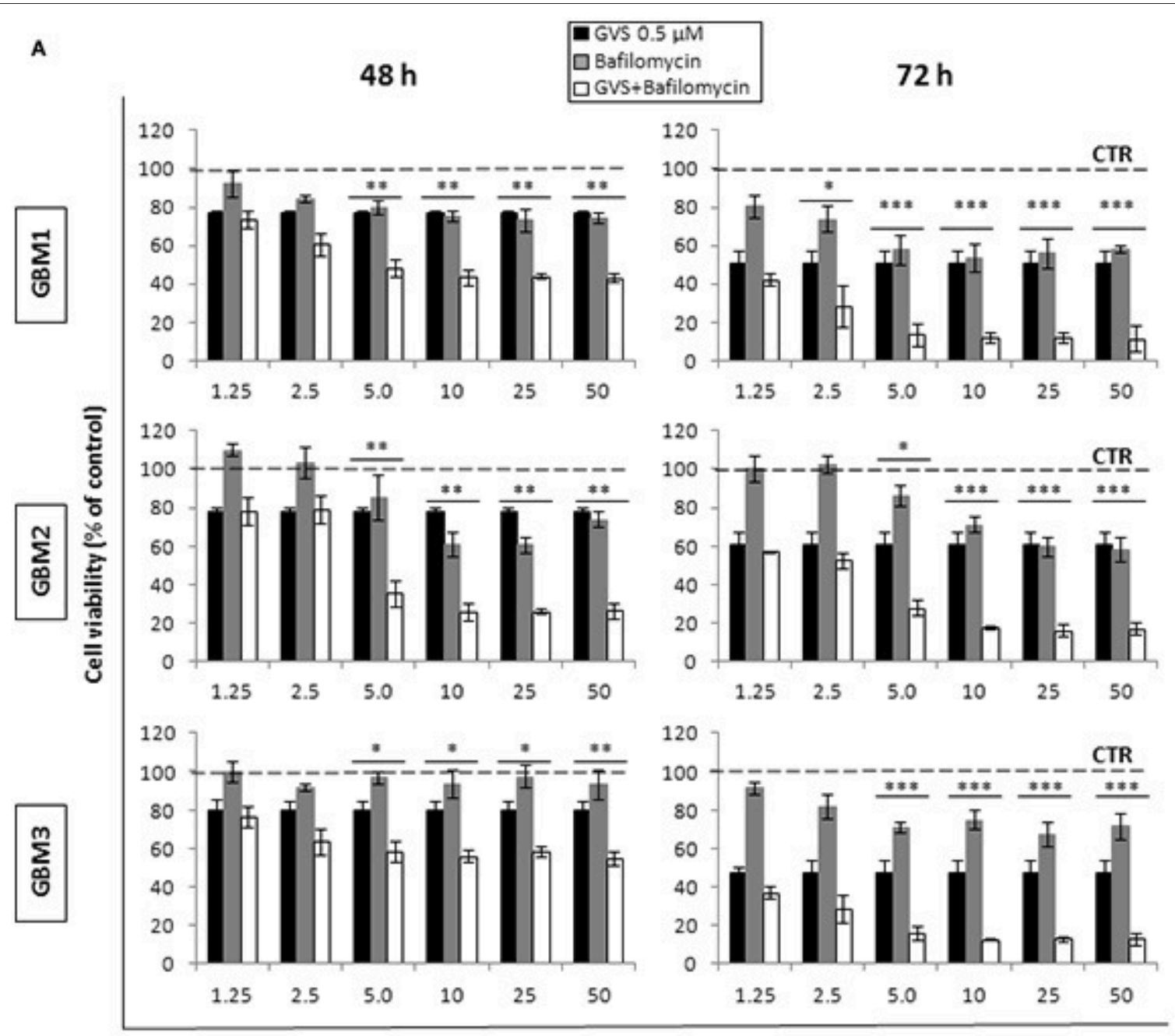

Bafilomycin (nM)

B

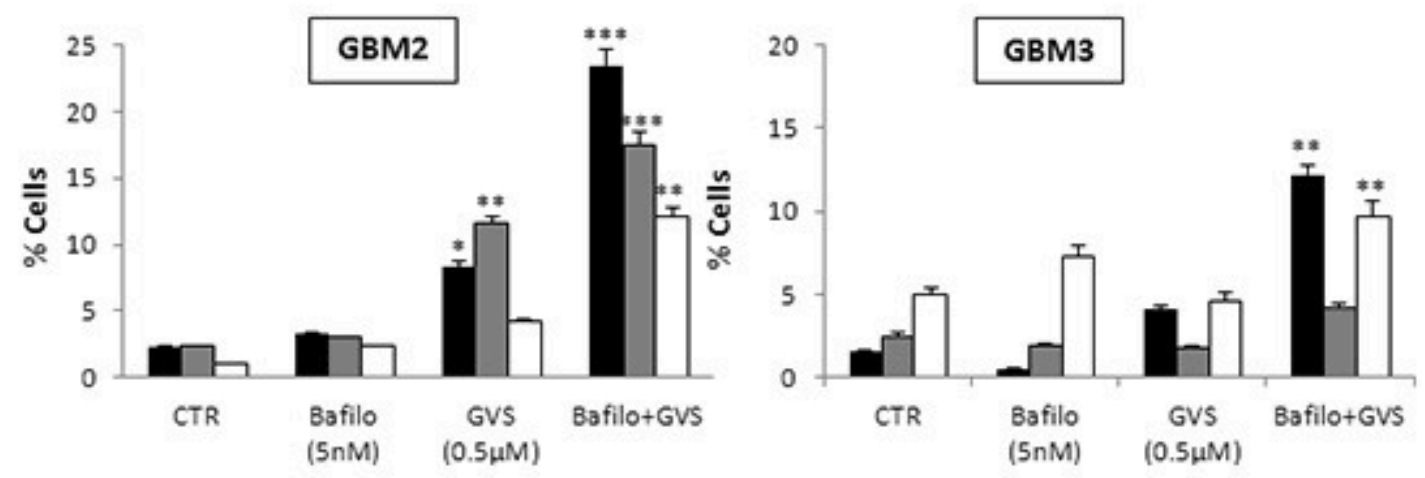

- Early apoptosis $n$ Late apoptosis $\square$ Necrosis

FIGURE 7 | Evaluation of the role of autophagy in GVS inhibitory effect on GBM CSCs. (A) GBM1, GBM2 and GBM3 CSCs were treated with GVS $0.5 \mu M$ (black bars), bafilomycin-A1 1.25, 2.5, 5, 10, 25, and $50 \mathrm{nM}$ (gray bars) or with the combination of all doses of the two drugs (pre-treatment with bafilomycin-A1 for 2 $\mathrm{h}$; white bars) for 48 and $72 \mathrm{~h}$. Cell viability was monitored by MTT assay. Statistical significance was calculated using ANOVA followed by Dunnett's post-hoc test; $\left({ }^{*} p<0.05,{ }^{* \star} p<0.01 ;{ }^{* \star *} p<0.001\right.$, vs. control). (B) In vitro assessment of apoptosis in GBM2 and GBM3 cells treated with bafilomycin-A1 (5 nM) or GVS (0.5 $\left.\mu \mathrm{M}\right)$, alone or in combination. FACS analysis of Annexin V-PI staining was used to measure apoptosis/necrosis. Histograms represent the percentage of each cell population after $48 \mathrm{~h}$ of drug exposure. Statistical analysis was performed using ANOVA followed by Dunnett's post-hoc test $\left({ }^{\star} p<0.05,{ }^{* *} p<0.01,{ }^{\star \star \star} p<0.0001\right.$ vs. control). 


\section{A}
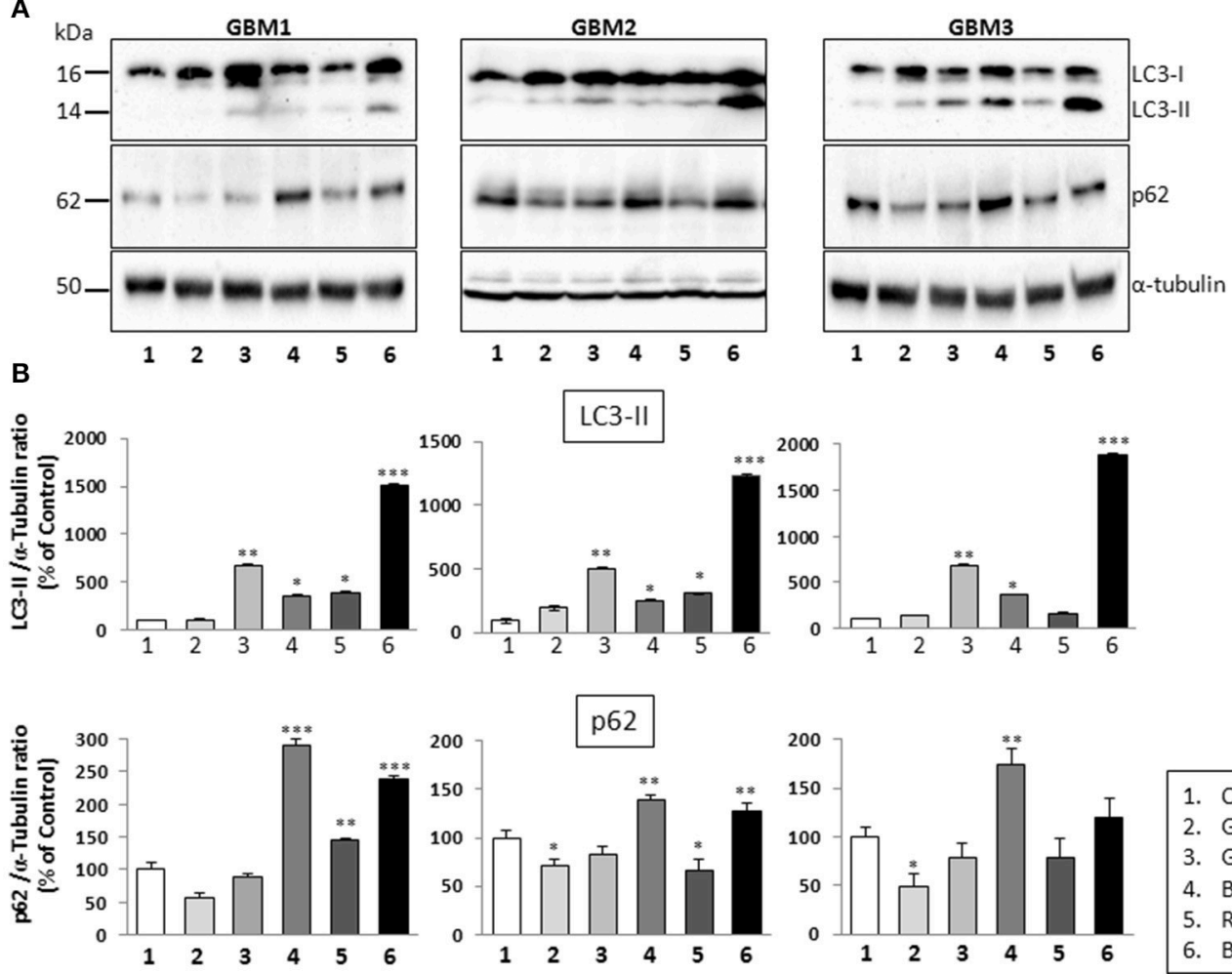

1. CTR

2. $\operatorname{GVS}(0.5 \mu \mathrm{M})$

3. $\operatorname{GVS}(1 \mu \mathrm{M})$

4. $\operatorname{BAF}(5 \mathrm{nM})$

5. RAPA $(40 \mathrm{nM})$

6. $B A F+G V S(0.5 \mu M)$

FIGURE 8 | Effect of the combined treatment with GVS and bafilomycin-A1 on the LC3-II and p62 protein levels. (A) Representative immunoblots of LC3-II and p62 protein levels. Cell lysates were obtained from GBM1, GBM2, and GBM3 CSCs treated with GVS (0.5-1.0 $\mu$ M), bafilomycin-A1 (5 nM), rapamycin (40 nM), and with GVS $0.5 \mu \mathrm{M} /$ bafilomycin-A1 combination (2 h pre-treatment with bafilomycin-A1). $\alpha$-tubulin content was used as loading control. (B) Densitometric analysis of LC3-II and p62 protein levels. Data were normalized against $\alpha$-tubulin and expressed as percentage of untreated control values. Statistical analysis was performed using ANOVA followed by Dunnett's post-hoc test ( ${ }^{*} p<0.05,{ }^{* \star} p<0.01 ;{ }^{* \star *} p<0.001$ vs. control) comparing GVS, bafilomycin-A1, rapamycin, GVS+ bafilomycin-A1 treatments to the respective untreated (CTR) GBM CCSs.

the capability of GVS to modify the autophagy process in CSCs, measuring the expression of main ATG proteins. In particular, GVS treatment produced increasing amount of key autophagy executory proteins LC3-II and Beclin1, clearly suggesting that the drug administration induced marked differences within the autophagy process. To further confirm this result, the effect of GVS on autophagy was also assessed by autophagosome analysis. Autophagosomes express LC3-II on the outer membranes, while LC3-I isoform, originated by different post-translational modifications, has a typical cytoplasmic diffuse expression pattern. GVS treatment of GBM CSCs, previously transduced with LC3-GFP baculovirus, induced a significant accumulation of discrete fluorescent LC3-GFP puncta, likely representing autophagosomes. Moreover, increased levels of LC3-GFP in the presence of GVS or compounds interfering with the autophagosome-lysosome fusion (i.e., bafilomycin-A1), was indicative of an increase of the synthesis of autophagy-related membranes (autophagy induction) rather than a reduction in vesicle clearance as for autophagy blockage (Klionsky et al., 2016). Moreover, transmission electron microscopy ultrastructural evaluation highlighted a marked increase in autophagy-like vesicles after GVS exposure. Altogether, these molecular and cellular features indicate that GVS treatment induces the activation of autophagy in GBM CSCs.

As widely discussed (Choi et al., 2013; Macintosh and Ryan, 2013; White, 2015), the contradictory role of autophagy in cancer makes difficult to understand whether this mechanism is per se beneficial or inhibitory for the malignant progression. The possible dual functional role of autophagy, i.e., promoting programmed cell death fate or conferring a pro-survival favorable phenotype to the cancer cell, can determine the success or failure of anti-cancer therapies (Palumbo and Comincini, 2013). This opposite behavior represents an adaptive response of the tumor cells to the therapy, and is associated to several tumor-environmental conditions, such as specific or off-targets effects of the drug used, tumor cell type and the state of stem-like or differentiated cells (Galluzzi et al., 2015). To decipher the contribution of the autophagy process to the cytotoxic effects of GVS, autophagy in GBM CSCs was positively or negatively modulated using different experimental approaches. To obtain the autophagy inhibition: the downregulation of key genes and/or proteins and the blockade of the autophagy flux preventing the fusion of autophagosomes with lysosomes; to activate the autophagy process: rapamycin 


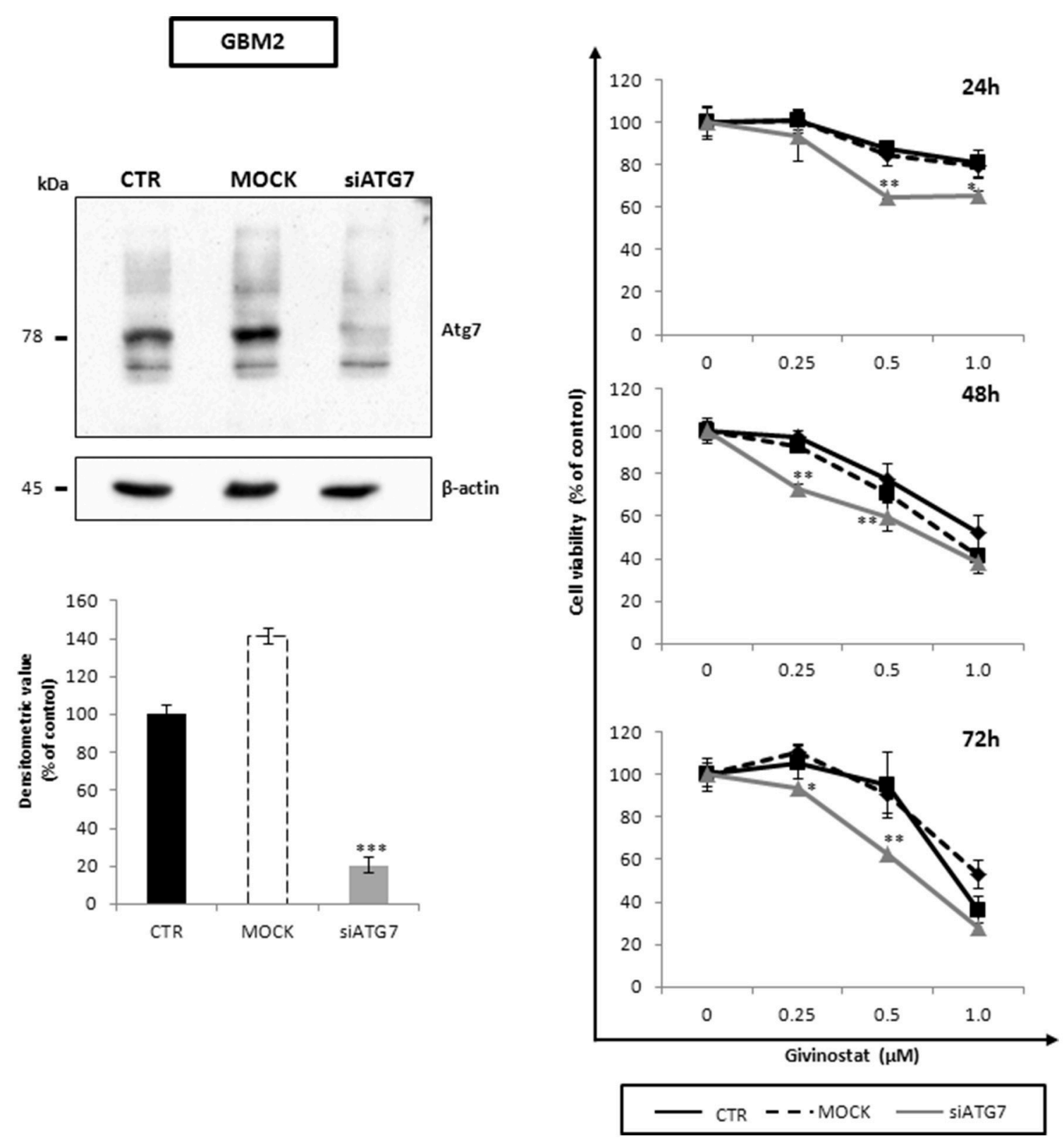

FIGURE 9 | Atg7 silencing effects on cell viability along with GVS in GBM CSCs. Equal amount of proteins obtained from GBM2 control (CTR), GBM2 transfected without siATG7 (MOCK) and GBM2 transfected with siATG7 were assayed. Immunoblotting analysis (left panels) was performed, $24 \mathrm{~h}$ post-transfection, in order to ensure ATG7 silencing and Atg7 downregulation. $\beta$-actin was used as loading control. MTT cell viability assay (right panels) was performed at 24,48 , and 72 h after GVS administration $(0.25,0.5$, and $1.0 \mu \mathrm{M})$, in CTR, MOCK and siATG7 transfected GBM2 cells. Statistical analysis was performed using ANOVA followed by Dunnett's post-hoc test $\left({ }^{*} p<0.05,{ }^{* *} p<0.01 ;{ }^{* \star *} p<0.001\right.$ vs. control).

treatment or growing cells in medium deprived of growth factors.

Bafilomycin-A1, used to inhibit the autophagy flux, is a specific inhibitor of the late phase of autophagy that prevents the acidification of the autophagic vacuoles and inhibits the fusion between autophagosomes and lysosomes (Yamamoto et al., 1998). In parallel, the inhibition of autophagy was achieved by down-regulating ATG7 gene by siRNA. Silencing ATG7, or other key autophagy genes, provides an effective strategy to arrest this pathway blocking the autophagosomes formation and the degradation processes (Saiki et al., 2011; Criollo et al., 2012; Klionsky et al., 2016). In this way, we show that the inhibition of autophagy by both molecular and pharmacological approaches, increases the anti-proliferative and pro-apoptotic effects of GVS on GBM CSCs. This result was confirmed in cells in which autophagy was activated by deprivation of growth factors. The 


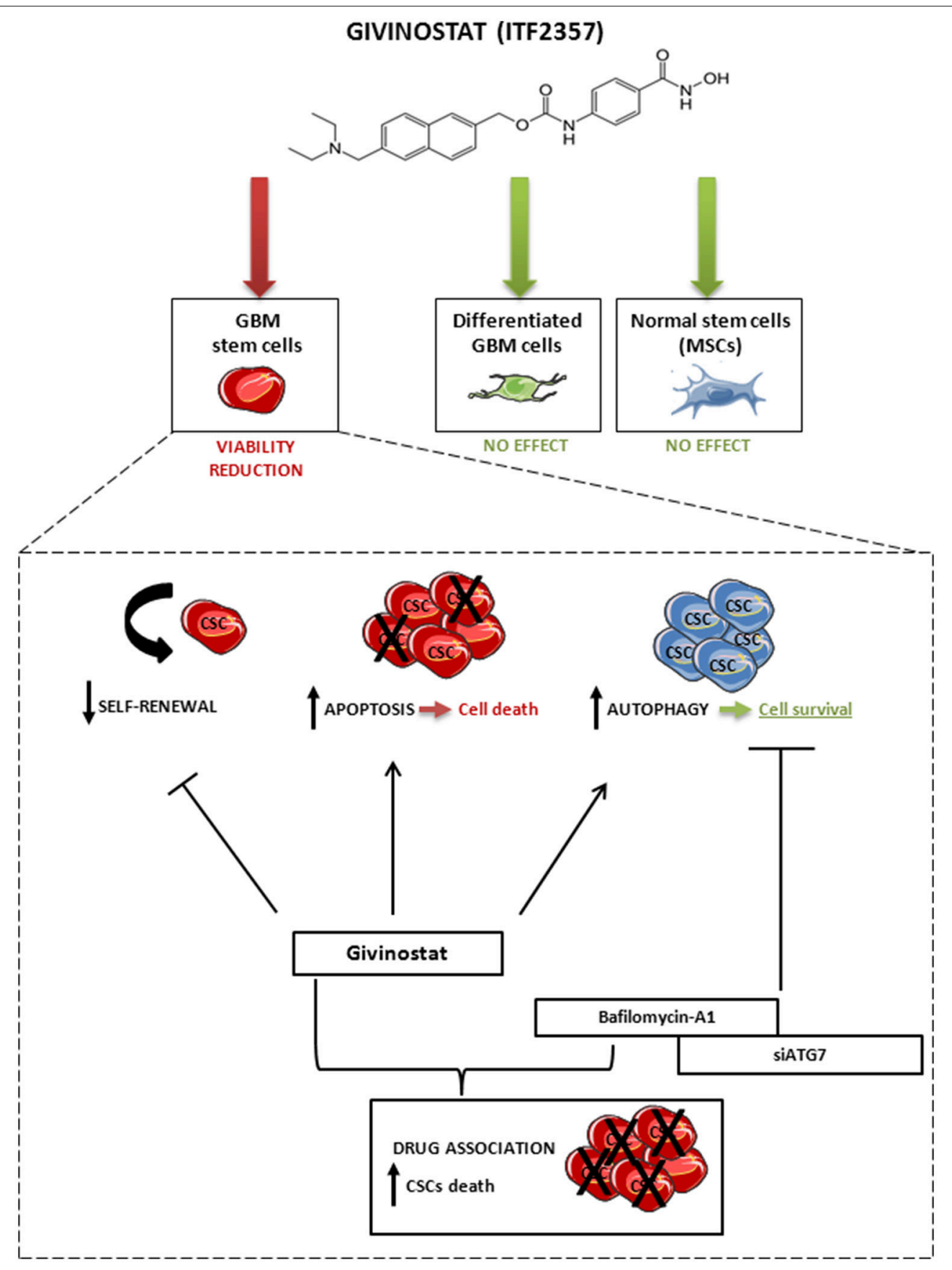

FIGURE 10 | Schematic representation of the effect of GVS on GBM CSCs, differentiated GBM cells and MSCs. GVS reduced CSC viability through apoptosis activation and self-renewal impairment; at the same time, GVS induced activation of the autophagy process that resulted in a partial protection of CSCs from GVS pharmacological activity. The autophagy flux arrest, mediated by bafilomycin-A1 co-administration or by ATG7 silencing, enhanced the GVS pro-apoptotic activity, therefore suggesting a pro-survival effect of the autophagic process to counteract the effect of GVS. Importantly, GVS did not reduce cells viability in differentiated GBM cells and MSCs.

depletion of nutrients is one of the main stimuli triggering autophagy (Mizushima and Klionsky, 2007). When the nutrients' supply is limited, cells activate autophagy to generate a source of metabolic substrates to sustain the energetic requirement for survival (Kang and Avery, 2008). We report that deprivation of growth factors in CSCs and the consequent activation of autophagy results in a protective effect against GVS antitumor activity.

Taken together, these findings suggest that the activation of autophagy occurring during GVS treatment has a cytoprotective 
effect that, supporting CSC survival, reduces HDACi efficacy. In other terms, tumor cells respond to GVS-induced cell death with the activation of autophagy, a process directed to catabolize damaged proteins and organelles. Lastly, our data indicate that combined treatment with GVS followed by autophagy inhibition has strikingly synergistic anti-tumor activity in GBM, similarly to lung (Del Bufalo et al., 2014), hepatoma (Liu et al., 2010; Yuan et al., 2014), breast (Rao et al., 2012) cancer models. Consistent with these data, we propose a mechanistic evaluation of the possible synergism between GVS effects and autophagy inhibition that enhances the loss of GBM CSC viability (Figure 10).

On the other hand, our results differ from previous studies showing that the combined treatment with imipramine and ticlopidine elicits cell-lethal autophagy in mouse models of gliomagenesis (Shchors et al., 2015). However, it must be pointed out that the quantitatively and qualitatively different modulations of autophagy in our and in the previous study might result in either pro-survival or cytotoxic effects (White et al., 2015).

In conclusion, we demonstrate that GVS is a promising pharmacological agent; its inhibitory activity against CSC proliferation and self-renewal, accomplished with high potency and efficacy, allows us to consider GVS as a novel possible adjuvant approach for GBM treatment. The identification of GVS as a drug specifically directed against CSCs could represent a significant pharmacological alternative for GBM patients. Moreover, our results suggest that, in light of the cellular and biological complexity of GBM, GVS therapeutic efficacy could be intensified by the association with autophagy inhibitors, since these compounds could synergize and revert the resistance to therapeutic interventions of CSCs, and reduce the drug concentration required to achieve a significant tumor mass reduction.

\section{AUTHOR CONTRIBUTIONS}

Conceived and designed the experiments: SC, TF, FB, FA, and GF. Performed the experiments: FA, AP, RW, AD, SC, and AS. Analyzed the data: all the authors. Contributed reagents/materials/analysis tools: SC, TF, and GF. Wrote the manuscript: FA, TF, SC, and GF.

\section{ACKNOWLEDGMENTS}

This work was supported by a grant from Italian Association from cancer Research (AIRC) to TF. The authors thank Italfarmaco S.p.A. for supplying ITF2357 and for the financial support. GF is an employee of Italfarmaco S.p.A.

\section{SUPPLEMENTARY MATERIAL}

The Supplementary Material for this article can be found online at: http://journal.frontiersin.org/article/10.3389/fnmol. 2016.00107/full\#supplementary-material
Supplementary Figure 1 | GVS dose-response curves (0.1-2 $\mu \mathrm{M})$ on U87-MG, U138-MG, and T98G cell viability. Every $24 \mathrm{~h}$, cell viability was determined by MTT assay for up to $96 \mathrm{~h}$. Experiments were performed in triplicate and the percentage of viability was calculated vs. untreated control cell. Statistical analysis was performed with ANOVA test followed by Dunnett's post-hoc test $\left(^{*} p\right.$ $<0.05,{ }^{* *} p<0.01,{ }^{* * *} p<0.001$ ).

Supplementary Figure 2 | Trypan blue exclusion assay on GBM1 and GBM2 CSCs. Analysis was performed after GVS $(0.5,1,2 \mu \mathrm{M})$ exposure from 48 to $72 \mathrm{~h}$ (black columns represent cells number at $\mathrm{T}_{0}$ ). Data represent the mean and the SD of three independent replicates; (for dead cells: * $p<0.05$, ${ }^{* *} p<$ $0.01,{ }^{* *} p<0.001$, by ANOVA test followed by Dunnett's post-hoc test).

Supplementary Figure 3 | Schematic representation of the experimental plan of time-scale cell growth recovery test.

Supplementary Figure 4 | Cell growth recovery assay performed on GBM1, GBM2, and GBM3 CSCs. Cells were treated with GVS $(0.5 \mu \mathrm{M})$, for 24,48 , and $72 \mathrm{~h}$. After each time of exposure, culture medium was replaced with a fresh one devoid of GVS and cell viability was tested by MTT assay at $T_{0}$ (time of medium replacement), and after 24, 48, and $72 \mathrm{~h}$. Histograms indicate the percentage of cell survival compared to untreated control value at $\mathrm{T}_{0}\left({ }^{*} p<0.05 ;{ }^{* *} p<0.01\right.$, ${ }^{* * *} p<0.001$, on ANOVA test followed by Dunnett's post-hoc test).

Supplementary Figure 5 | GVS dose-response curves performed on (A) differentiated GBM1 and GBM2 CSCs and (B) human umbilical cord-derived MSCs. Cell viability was tested after 24-144 h of GVS treatment $(0.1-2 \mu \mathrm{M})$ and was determined by MTT assay. Experiments were performed in triplicate and percentage of inhibition was calculated vs. untreated control. Statistical analysis was performed with ANOVA test followed by Dunnett's post-hoc test $\left({ }^{*} p<0.05,{ }^{* *} p<0.01\right)$.

Supplementary Figure 6 | MAP1LC3B expression in GBM1, GBM2, and GBM3 CSCs. Cells were treated with GVS $(1 \mu \mathrm{M})$ for 24,48 , and $72 \mathrm{~h}$ and assayed for MAP1LC3B mRNA levels by Real time qPCR. Results are given as relative mRNA expression, in arbitrary units of the ratio of the target RNA over HPRT1 and TBP expression levels. Statistical analysis was performed with ANOVA test followed by Dunnett's post-hoc test. Bars represent the mean of three independent experiments $\pm \mathrm{SD}\left({ }^{*} p<0.05 ;{ }^{* *} p<0.01\right)$.

Supplementary Figure 7 | CompuSyn software evaluation of the synergistic effect of GVS in combination with bafilomycin-A1 in GBM CSCs. Isobolograms of drug combination on GBM1, GBM2, and GBM3 CSC viability after treatment for 48 and $72 \mathrm{~h}$, are represented. Combination index $(\mathrm{Cl})$ is represented by symbols above (indicate antagonism between drugs) or below the line (indicate synergy) and in the Table on the right.

Supplementary Figure 8 | Effect of deprivation of growth factors on GVS activity in GBM CSCs. GBM1 and GBM2 CSCs were maintained in the absence of growth factors for $60 \mathrm{~h}$; after this period cells were treated with GVS $(0.1,0.25$, $0.5,1.0$, and $2.0 \mu \mathrm{M})$ for further $48 \mathrm{~h}$ and viability was assessed by MTT assay. In parallel the same study was performed on GBM1 and GBM2 maintained in complete stem medium. Statistical analysis was performed with unpaired two-tailed $t$-test $\left.{ }^{*} p<0.05,{ }^{* *} p<0.01 ; * * * p<0.001\right)$. To confirm that deprivation of growth factors really increases autophagy, immunoblotting analysis was performed on cell lysates. LC3-I, LC3-II, and p62 protein levels were assayed (right panels).

Supplementary Table 1 | Main clinical-pathological features of tumors, and tumorigenic potential in mice of GBM-derived cell cultures enriched in CSCs.

Supplementary Table 2 | Inhibition percentage value and statistical significance of GVS antiproliferative effect on GBM CSCs. Data were obtained from mean percentage of cell viability of treated cells vs. untreated control cells for each concentration and time point of GVS exposure. Statistical analysis was performed with ANOVA test followed by Dunnett's post-hoc test; ${ }^{*} p<0.05,{ }^{* *} p<0.01,{ }^{* * *} p<0.001$ (NS, non-significant; blank boxes, not performed). 


\section{REFERENCES}

Al-Hajj, M., Becker, M. W., Wicha, M., Weissman, I., and Clarke, M. F. (2004). Therapeutic implications of cancer stem cells. Curr. Opin. Genet. Dev. 14, 43-47. doi: 10.1016/j.gde.2003.11.007

Armeanu, S., Pathil, A., Venturelli, S., Mascagni, P., Weiss, T. S., Gottlicher, M., et al. (2005). Apoptosis on hepatoma cells but not on primary hepatocytes by histone deacetylase inhibitors valproate and ITF2357. J. Hepatol. 42, 210-217. doi: 10.1016/j.jhep.2004.10.020

Bajetto, A., Porcile, C., Pattarozzi, A., Scotti, L., Aceto, A., Daga, A., et al. (2013). Differential role of EGF and BFGF in human GBM-TIC proliferation: relationship to EGFR-tyrosine kinase inhibitor sensibility. J. Biol. Regul. Homeost. Agents 27, 143-154.

Banelli, B., Carra, E., Barbieri, F., Wurth, R., Parodi, F., Pattarozzi, A., et al. (2015). The histone demethylase KDM5A is a key factor for the resistance to temozolomide in glioblastoma. Cell Cycle 14, 3418-3429. doi: 10.1080/15384101.2015.1090063

Bolden, J. E., Shi, W., Jankowski, K., Kan, C. Y., Cluse, L., Martin, B. P., et al. (2013). HDAC inhibitors induce tumor-cell-selective pro-apoptotic transcriptional responses. Cell Death Dis. 4:e519. doi: 10.1038/cddis.2013.9

Bonavia, R., Inda, M. M., Cavenee, W. K., and Furnari, F. B. (2011). Heterogeneity maintenance in glioblastoma: a social network. Cancer Res. 71, 4055-4060. doi: 10.1158/0008-5472.CAN-11-0153

Brennan, C. W., Verhaak, R. G., Mckenna, A., Campos, B., Noushmehr, H., Salama, S. R., et al. (2013). The somatic genomic landscape of glioblastoma. Cell 155, 462-477. doi: 10.1016/j.cell.2013.09.034

Carra, E., Barbieri, F., Marubbi, D., Pattarozzi, A., Favoni, R. E., Florio, T., et al. (2013). Sorafenib selectively depletes human glioblastoma tumorinitiating cells from primary cultures. Cell Cycle 12, 491-500. doi: 10.4161/cc. 23372

Chen, J., Li, Y., Yu, T. S., Mckay, R. M., Burns, D. K., Kernie, S. G., et al. (2012). A restricted cell population propagates glioblastoma growth after chemotherapy. Nature 488, 522-526. doi: 10.1038/nature11287

Choi, A. M. K., Ryter, S. W., and Levine, B. (2013). Autophagy in human health and disease. New Engl. J. Med. 368, 651-662. doi: 10.1056/NEJMra1205406

Chou, T. C. (2010). Drug combination studies and their synergy quantification using the Chou-Talalay method. Cancer Res. 70, 440-446. doi: 10.1158/00085472.CAN-09-1947

Chou, T. C., and Talalay, P. (1984). Quantitative analysis of dose-effect relationships: the combined effects of multiple drugs or enzyme inhibitors. $A d v$. Enzyme Regul. 22, 27-55. doi: 10.1016/0065-2571(84)90007-4

Corsaro, A., Bajetto, A., Thellung, S., Begani, G., Villa, V., Nizzari, M., et al. (2016). Cellular prion protein controls stem cell-like properties of human glioblastoma tumor-initiating cells. Oncotarget 7, 38638-38657. doi: 10.18632/oncotarget.9575

Criollo, A., Chereau, F., Malik, S. A., Niso-Santano, M., Marino, G., Galluzzi, L., et al. (2012). Autophagy is required for the activation of NFkB. Cell Cycle 11, 194-199. doi: 10.4161/cc.11.1.18669

Del Bufalo, D., Desideri, M., De Luca, T., Di Martile, M., Gabellini, C., Monica, V., et al. (2014). Histone deacetylase inhibition synergistically enhances pemetrexed cytotoxicity through induction of apoptosis and autophagy in non-small cell lung cancer. Mol. Cancer 13:230. doi: 10.1186/1476-459 8-13-230

De Souza, C., and Chatterji, B. P. (2015). HDAC inhibitors as novel anticancer therapeutics. Recent Pat. Anticancer Drug Discov. 10, 145-162. doi: $10.2174 / 1574892810666150317144511$

Dominici, M., Le Blanc, K., Mueller, I., Slaper-Cortenbach, I., Marini, F. C., Krause, D. S., et al. (2006). Minimal criteria for defining multipotent mesenchymal stromal cells. The international society for cellular therapy position statement. Cytotherapy 8, 315-317. doi: 10.1080/14653240600855905

Fassina, L., Magenes, G., Inzaghi, A., Palumbo, S., Allavena, G., Miracco, C., et al. (2012). AUTOCOUNTER, an Image JavaScript to analyze LC3BGFP expression dynamics in autophagy-induced astrocytoma cells. Eur. J. Histochem. 56:e44. doi: 10.4081/ejh.2012.e44

Favoni, R. E., Pattarozzi, A., Lo Casto, M., Barbieri, F., Gatti, M., Paleari, L., et al. (2010). Gefitinib targets EGFR dimerization and ERK1/2 phosphorylation to inhibit pleural mesothelioma cell proliferation. Curr. Cancer Drug Targets 10, 176-191. doi: 10.2174/156800910791054130
Florio, T., and Barbieri, F. (2012). The status of the art of human malignant glioma management: the promising role of targeting tumor-initiating cells. Drug Discov. Today 17, 1103-1110. doi: 10.1016/j.drudis.2012.06.001

Galimberti, S., Canestraro, M., Savli, H., Palumbo, G. A., Tibullo, D., Nagy, B., et al. (2010). ITF2357 interferes with apoptosis and inflammatory pathways in the HL-60 model: a gene expression study. Anticancer Res. 30, 4525-4535.

Galluzzi, L., Pietrocola, F., Bravo-San Pedro, J. M., Amaravadi, R. K., Baehrecke, E. H., Cecconi, F., et al. (2015). Autophagy in malignant transformation and cancer progression. EMBO J. 34, 856-880. doi: 10.15252/embj.201 490784

Gatti, M., Pattarozzi, A., Bajetto, A., Würth, R., Daga, A., Fiaschi, P., et al. (2013). Inhibition of CXCL12/CXCR4 autocrine/paracrine loop reduces viability of human glioblastoma stem-like cells affecting self-renewal activity. Toxicology 314, 209-220. doi: 10.1016/j.tox.2013.10.003

Golay, J., Cuppini, L., Leoni, F., Micò, C., Barbui, V., Domenghini, M., et al. (2007). The histone deacetylase inhibitor ITF2357 has anti-leukemic activity in vitro and in vivo and inhibits IL-6 and VEGF production by stromal cells. Leukemia 21, 1892-1900. doi: 10.1038/sj.leu.2404860

Griffero, F., Daga, A., Marubbi, D., Capra, M. C., Melotti, A., Pattarozzi, A., et al. (2009). Different response of human glioma tumor-initiating cells to epidermal growth factor receptor kinase inhibitors. J. Biol. Chem. 284, 7138-7148. doi: 10.1074/jbc.M807111200

Gritti, M., Würth, R., Angelini, M., Barbieri, F., Peretti, M., Pizzi, E., et al. (2014). Metformin repositioning as antitumoral agent: selective antiproliferative effects in human glioblastoma stem cells, via inhibition of CLIC1-mediated ion current. Oncotarget 5, 11252-11268. doi: 10.18632/oncotarget.2617

Haggarty, S. J., Koeller, K. M., Wong, J. C., Grozinger, C. M., and Schreiber, S. L. (2003). Domain-selective small-molecule inhibitor of histone deacetylase 6 (HDAC6)-mediated tubulin deacetylation. Proc. Natl. Acad. Sci. U.S.A. 100, 4389-4394. doi: 10.1073/pnas.0430973100

Kang, C., and Avery, L. (2008). To be or not to be, the level of autophagy is the question: dual roles of autophagy in the survival response to starvation. Autophagy 4, 82-84. doi: 10.4161/auto.5154

Klionsky, D. J., Abdelmohsen, K., Abe, A., Abedin, M. J., Abeliovich, H., Acevedo Arozena, A., et al. (2016). Guidelines for the use and interpretation of assays for monitoring autophagy (3rd edition). Autophagy 12, 1-222. doi: $10.1080 / 15548627.2015 .1100356$

Lee, J., Kotliarova, S., Kotliarov, Y., Li, A., Su, Q., Donin, N. M., et al. (2006). Tumor stem cells derived from glioblastomas cultured in bFGF and EGF more closely mirror the phenotype and genotype of primary tumors than do serum-cultured cell lines. Cancer Cell 9, 391-403. doi: 10.1016/j.ccr.2006.03.030

Lee, P., Murphy, B., Miller, R., Menon, V., Banik, N. L., Giglio, P., et al. (2015). Mechanisms and clinical significance of histone deacetylase inhibitors: epigenetic glioblastoma therapy. Anticancer Res. 35, 615-625.

Leoni, F., Fossati, G., Lewis, E. C., Lee, J. K., Porro, G., Pagani, P., et al. (2005). The histone deacetylase inhibitor ITF2357 reduces production of pro-inflammatory cytokines in vitro and systemic inflammation in vivo. Mol. Med. 11, 1-15. doi: 10.2119/2006-00005.Dinarello

Liu, Y. L., Yang, P. M., Shun, C. T., Wu, M. S., Weng, J. R., and Chen, C. C. (2010). Autophagy potentiates the anti-cancer effects of the histone deacetylase inhibitors in hepatocellular carcinoma. Autophagy 6, 1057-1065. doi: 10.4161/auto.6.8.13365

Macintosh, R. L., and Ryan, K. M. (2013). Autophagy in tumour cell death. Semin. Cancer Biol. 23, 344-351. doi: 10.1016/j.semcancer.2013.05.006

Marchesi, N., Osera, C., Fassina, L., Amadio, M., Angeletti, F., Morini, M., et al. (2014). Autophagy is modulated in human neuroblastoma cells through direct exposition to low frequency electromagnetic fields. J. Cell. Physiol. 229, 1776-1786. doi: 10.1002/jcp.24631

Massa, A., Barbieri, F., Aiello, C., Arena, S., Pattarozzi, A., Pirani, P., et al. (2004). The expression of the phosphotyrosine phosphatase DEP-1/PTPeta dictates the responsivity of glioma cells to somatostatin inhibition of cell proliferation. $J$. Biol. Chem. 279, 29004-29012. doi: 10.1074/jbc.M403573200

Mathew, R., Karp, C. M., Beaudoin, B., Vuong, N., Chen, G., Chen, H. Y., et al. (2009). Autophagy suppresses tumorigenesis through elimination of p62. Cell 137, 1062-1075. doi: 10.1016/j.cell.2009.03.048

Mathur, D. (2014). Selection of suitable housekeeping genes for expression analysis in glioblastoma using quantitative RT-PCR. Ann. Neurosci. 21, 62-63. doi: 10.5214/ans.0972.7531.210207 
Mizushima, N., and Klionsky, D. J. (2007). Protein turnover via autophagy: implications for metabolism. Annu. Rev. Nutr. 27, 19-40. doi: 10.1146/annurev.nutr.27.061406.093749

Ohgaki, H., and Kleihues, P. (2013). The definition of primary and secondary glioblastoma. Clin. Cancer Res. 19, 764-772. doi: 10.1158/1078-0432.CCR-123002

Omuro, A., and Deangelis, L. M. (2013). Glioblastoma and other malignant gliomas: a clinical review. JAMA 310, 1842-1850. doi: 10.1001/jama.2013.280319

Palumbo, S., and Comincini, S. (2013). Autophagy and ionizing radiation in tumors: the "survive or not survive" dilemma. J. Cell. Physiol. 228, 1-8. doi: $10.1002 /$ jcp. 24118

Patel, A. P., Tirosh, I., Trombetta, J. J., Shalek, A. K., Gillespie, S. M., Wakimoto, H., et al. (2014). Single-cell RNA-seq highlights intratumoral heterogeneity in primary glioblastoma. Science 344, 1396-1401. doi: 10.1126/science.1254257

Pathil, A., Armeanu, S., Venturelli, S., Mascagni, P., Weiss, T. S., Gregor, M., et al. (2006). HDAC inhibitor treatment of hepatoma cells induces both TRAILindependent apoptosis and restoration of sensitivity to TRAIL. Hepatology 43, 425-434. doi: 10.1002/hep.21054

Pattarozzi, A., Gatti, M., Barbieri, F., Würth, R., Porcile, C., Lunardi, G., et al. (2008). 17beta-estradiol promotes breast cancer cell proliferationinducing stromal cell-derived factor-1-mediated epidermal growth factor receptor transactivation: reversal by gefitinib pretreatment. Mol. Pharmacol. 73, 191-202. doi: 10.1124/mol.107.039974

Pazolli, E., Alspach, E., Milczarek, A., Prior, J., Piwnica-Worms, D., and Stewart, S. A. (2012). Chromatin remodeling underlies the senescence-associated secretory phenotype of tumor stromal fibroblasts that supports cancer progression. Cancer Res. 72, 2251-2261. doi: 10.1158/0008-5472.CAN-11-3386

Peart, M. J., Smyth, G. K., van Laar, R. K., Bowtell, D. D., Richon, V. M., Marks, P. A., et al. (2005). Identification and functional significance of genes regulated by structurally different histone deacetylase inhibitors. Proc. Natl. Acad. Sci. U.S.A. 102, 3697-3702. doi: 10.1073/pnas.0500369102

Rabinowitz, J. D., and White, E. (2010). Autophagy and metabolism. Science 330, 1344-1348. doi: 10.1126/science.1193497

Rao, R., Balusu, R., Fiskus, W., Mudunuru, U., Venkannagari, S., Chauhan, L., et al. (2012). Combination of pan-histone deacetylase inhibitor and autophagy inhibitor exerts superior efficacy against triple-negative human breast cancer cells. Mol. Cancer Ther. 11, 973-983. doi: 10.1158/1535-7163.MCT-11-0979

Robert, T., Vanoli, F., Chiolo, I., Shubassi, G., Bernstein, K. A., Rothstein, R., et al. (2011). HDACs link the DNA damage response, processing of double-strand breaks and autophagy. Nature 471, 74-79. doi: 10.1038/nature09803

Saiki, S., Sasazawa, Y., Imamichi, Y., Kawajiri, S., Fujimaki, T., Tanida, I., et al. (2011). Caffeine induces apoptosis by enhancement of autophagy via PI3K/Akt/mTOR/p70S6K inhibition. Autophagy 7, 176-187. doi: 10.4161/auto.7.2.14074

Shchors, K., Massaras, A., and Hanahan, D. (2015). Dual targeting of the autophagic regulatory circuitry in gliomas with repurposed drugs elicits cell-lethal autophagy and therapeutic benefit. Cancer Cell 28, 456-471. doi: 10.1016/j.ccell.2015.08.012

Singh, S. K., Hawkins, C., Clarke, I. D., Squire, J. A., Bayani, J., Hide, T., et al. (2004). Identification of human brain tumour initiating cells. Nature 432, 396-401. doi: 10.1038/nature03128

Soeda, A., Inagaki, A., Oka, N., Ikegame, Y., Aoki, H., Yoshimura, S., et al. (2008). Epidermal growth factor plays a crucial role in mitogenic regulation of human brain tumor stem cells. J. Biol. Chem. 283, 10958-10966. doi: 10.1074/jbc.M704205200
Stupp, R., Mason, W. P., van Den Bent, M. J., Weller, M., Fisher, B., Taphoorn, M. J., et al. (2005). Radiotherapy plus concomitant and adjuvant temozolomide for glioblastoma. N. Engl. J. Med. 352, 987-996. doi: 10.1056/NEJMoa 043330

Thurn, K. T., Thomas, S., Moore, A., and Munster, P. N. (2011). Rational therapeutic combinations with histone deacetylase inhibitors for the treatment of cancer. Future Oncol. 7, 263-283. doi: 10.2217/fon.11.2

Ververis, K., and Karagiannis, T. C. (2012). Overview of the classical histone deacetylase enzymes and histone deacetylase inhibitors. ISRN Cell Biol. 2012:12. doi: $10.5402 / 2012 / 130360$

Vescovi, A. L., Galli, R., and Reynolds, B. A. (2006). Brain tumour stem cells. Nat. Rev. Cancer 6, 425-436. doi: 10.1038/nrc1889

Villa, V., Thellung, S., Corsaro, A., Novelli, F., Tasso, B., Colucci-D’amato, L., et al. (2016). Celecoxib inhibits prion protein 90-231-mediated pro-inflammatory responses in microglial cells. Mol. Neurobiol. 53, 57-72. doi: 10.1007/s12035014-8982-4

White, E. (2015). The role for autophagy in cancer. J. Clin. Invest. 125, 42-46. doi: 10.1172/JCI73941

White, E., Mehnert, J. M., and Chan, C. S. (2015). Autophagy, metabolism, and cancer. Clin. Cancer Res. 21, 5037-5046. doi: 10.1158/1078-0432.CCR-15-0490

Würth, R., Barbieri, F., and Florio, T. (2014). New molecules and old drugs as emerging approaches to selectively target human glioblastoma cancer stem cells. Biomed Res. Int. 2014:126586. doi: 10.1155/2014/126586

Würth, R., Barbieri, F., Pattarozzi, A., Gaudenzi, G., Gatto, F., Fiaschi, P., et al. (2016). Phenotypical and pharmacological characterization of stem-like cells in human pituitary adenomas. Mol. Neurobiol. doi: 10.1007/s12035-016-0025-x. [Epub ahead of print].

Würth, R., Pattarozzi, A., Gatti, M., Bajetto, A., Corsaro, A., Parodi, A., et al. (2013). Metformin selectively affects human glioblastoma tumor-initiating cell viability: a role for metformin-induced inhibition of Akt. Cell Cycle 12, 145-156. doi: 10.4161/cc.23050

Yamamoto, A., Tagawa, Y., Yoshimori, T., Moriyama, Y., Masaki, R., and Tashiro, Y. (1998). Bafilomycin A1 prevents maturation of autophagic vacuoles by inhibiting fusion between autophagosomes and lysosomes in rat hepatoma cell line, H-4-II-E cells. Cell Struct. Funct. 23, 33-42. doi: 10.1247/csf. 23.33

Yuan, H., Li, A. J., Ma, S. L., Cui, L. J., Wu, B., Yin, L., et al. (2014). Inhibition of autophagy signi fi cantly enhances combination therapy with sorafenib and HDAC inhibitors for human hepatoma cells. World J. Gastroenterol. 20, 4953-4962. doi: 10.3748/wjg.v20.i17.4953

Zhang, J., and Zhong, Q. (2014). Histone deacetylase inhibitors and cell death. Cell. Mol. Life Sci. 71, 3885-3901. doi: 10.1007/s00018-014-1656-6

Conflict of Interest Statement: GF is an employee of Italfarmaco S.p.A.

The other authors declare that the research was conducted in the absence of any commercial or financial relationships that could be construed as a potential conflict of interest.

Copyright (c) 2016 Angeletti, Fossati, Pattarozzi, Würth, Solari, Daga, Masiello, Barbieri, Florio and Comincini. This is an open-access article distributed under the terms of the Creative Commons Attribution License (CC BY). The use, distribution or reproduction in other forums is permitted, provided the original author(s) or licensor are credited and that the original publication in this journal is cited, in accordance with accepted academic practice. No use, distribution or reproduction is permitted which does not comply with these terms. 